\title{
Coherent acoustic interferometry during the photo-driven oxygen evolution reaction associates strain fields with the reactive oxygen intermediate (Ti-OH*)
}

Authors: Suryansh Singh ${ }^{\alpha, \gamma}$, Hanna Lyle ${ }^{\alpha, \gamma}$, Luca D’Amario ${ }^{\nu}$, Elena Magnano ${ }^{\chi}$, Ilya Vinogradov ${ }^{\alpha, *}$, and Tanja $\mathrm{Cuk}^{\alpha, \perp^{*}}$

\section{Affiliations:}

${ }^{\alpha}$ Renewable and Sustainable Energy Institute (RASEI), University of Colorado, Boulder, Boulder, 80303

${ }^{\gamma}$ Materials Science and Engineering Program, University of Colorado, Boulder, Boulder, 80303

${ }^{\vee}$ Department of Chemistry-Ångström Laboratories, Uppsala University, Box 523, SE75120 Uppsala, Sweden, and Department of Physics, Freie Universität Berlin, Arnimallee 14, 14195 Berlin, Germany

¿IOM CNR laboratorio TASC, $34149 \quad$ Basovizza $\quad$ (TS), Italy and Department of Physics, University of Johannesburg, PO Box 524, Auckland Park 2006, South Africa

${ }^{\perp}$ Department of Chemistry, University of Colorado, Boulder, Boulder, 80303

*Corresponding Authors: tanja.cuk@colorado.edu, ilya.vinogradov@colorado.edu

\begin{abstract}
:
The oxygen evolution reaction (OER) from water requires the formation of meta-stable, reactive oxygen intermediates to enable oxygen-oxygen bond formation. On the other hand, such reactive intermediates could also structurally modify the catalyst. A descriptor for the overall catalytic activity, the first electron and proton transfer OER intermediate from water, $\left(\mathrm{M}-\mathrm{OH}^{*}\right)$, has been associated with significant distortions of the metal-oxygen bonds upon charge-trapping. Time-resolved spectroscopy of in-situ, photo-driven OER on transition metal oxide surfaces has characterized $\mathrm{M}-\mathrm{OH}^{*}$ for the charge trapped and the symmetry of the lattice distortions by optical and vibrational transitions, respectively, but had yet to detect an interfacial strain field arising from a surface coverage $\mathrm{M}-\mathrm{OH}^{*}$. Here, we utilize picosecond, coherent acoustic interferometry to detect the uniaxial strain normal (100) to the $\mathrm{SrTiO}_{3} /$ aqueous interface directly caused by $\mathrm{Ti}-\mathrm{OH}^{*}$. The spectral analysis applies a fairly general methodology for detecting a combination of the spatial extent, magnitude, and generation time of the interfacial strain through the coherent oscillations'
\end{abstract}


phase. For lightly n-doped $\mathrm{SrTiO}_{3}$, we identify the strain generation time (1.31 ps), which occurs simultaneously with $\mathrm{Ti}^{-} \mathrm{OH}^{*}$ formation, and a tensile strain of $0.06 \%$ (upper limit $0.6 \%$ ). In addition to fully characterizing this intermediate across visible, mid-infrared, and now $\mathrm{GHz}-\mathrm{THz}$ probes on $\mathrm{SrTiO}_{3}$, that strain fields occur with the creation of some $\mathrm{M}-\mathrm{OH}^{*}$ modifies design strategies for tuning material properties for catalytic activity and provides insight into photoinduced degradation so prevalent for OER. To that end, the work put forth here provides a unique methodology to characterize intermediate-induced interfacial strain across OER catalysts.

\section{Introduction}

Meta-stable intermediates guide the oxygen evolution reaction (OER) from water, and are characterized by a series of electron and proton transfers from a metal-oxide surface. ${ }^{1-3}$ Often, the first electron and proton transfer from a water adsorbed site is utilized to classify materials' catalytic activity for OER. ${ }^{4,5}$ For example, a too strong or weak binding of oxygen to the metal site upon this electron and proton transfer correlates with lower catalytic activity, ${ }^{5}$ with strong binding implying that $\mathrm{O}-\mathrm{O}$ bond formation rate limits the reaction and with weak binding implying that creating the first intermediate does. While this reactive oxygen intermediate $\left(\mathrm{M}-\mathrm{OH}^{*}\right)$ is often compared energetically between materials ${ }^{1}$ by DFT calculations with limited surface relaxation allowed, ${ }^{6}$ its structural form has only recently been elucidated in a series of in-situ and timeresolved experiments using optical ${ }^{7-11}$ and vibrational ${ }^{12-15}$ spectroscopy. Significant distortions of the metal-oxygen bond in $\mathrm{M}-\mathrm{OH}^{*}$ are evidenced by, upon charge-trapping, a double bond on $\mathrm{Fe}_{2} \mathrm{O}_{3}$ $(\mathrm{Fe}=\mathrm{O})^{15}$ and $\mathrm{Co}_{3} \mathrm{O}_{4}(\mathrm{Co}=\mathrm{O})^{13}$ and a lengthened Ti-O bond on $\mathrm{SrTiO}_{3} .{ }^{12}$ This is not unanticipated by calculations of charge-trapping on surfaces of bulk semiconductors: ${ }^{16-18}$ when charge traps in periodic lattices, lattice deformations form around the carrier to create a "polaron" that then transports charge with the accompanying distortions. Hole-polarons are especially ubiquitous in $3 \mathrm{~d}$ transition metal oxides ${ }^{19}$ due to the susceptibility of the $3 \mathrm{~d}$ M-O $2 \mathrm{p}$ octahedral bonding environment to local deformations such as the Jahn-Teller effect. In regard to catalysis at surfaces, these local deformations can define "active sites" such as that suggested by the structural characterization of $\mathrm{M}-\mathrm{OH}^{*}$.

On the other hand, surfaces of transition metal oxides are associated with strain fields at the vacuum and liquid interfaces..$^{20,21}$ Strain engineering has been utilized to modify the ground state properties of these catalysts, especially within the perovskite families. In-plane compressive 
and tensile strains modify the adsorption energy of oxygen, the d-band width, and oxygen diffusion, ${ }^{20,22}$ with some associations to OER activity; bulk strain also modifies these properties. ${ }^{22}$ Further, interfacial strain is invoked in describing catalysts' surface re-structuring under potential or light driven conditions. In electrochemistry, Pourbaix diagrams define a surfaces' stability against $\mathrm{pH}$ and potential, ${ }^{23-26}$ leading to new structural phases very close to the noted stable region of OER on the catalyst and metal leaching into the electrolyte. In recent solar-to-fuel devices, photo-driven degradation often exacerbates the problem of finding an active and stable catalyst. ${ }^{27}$
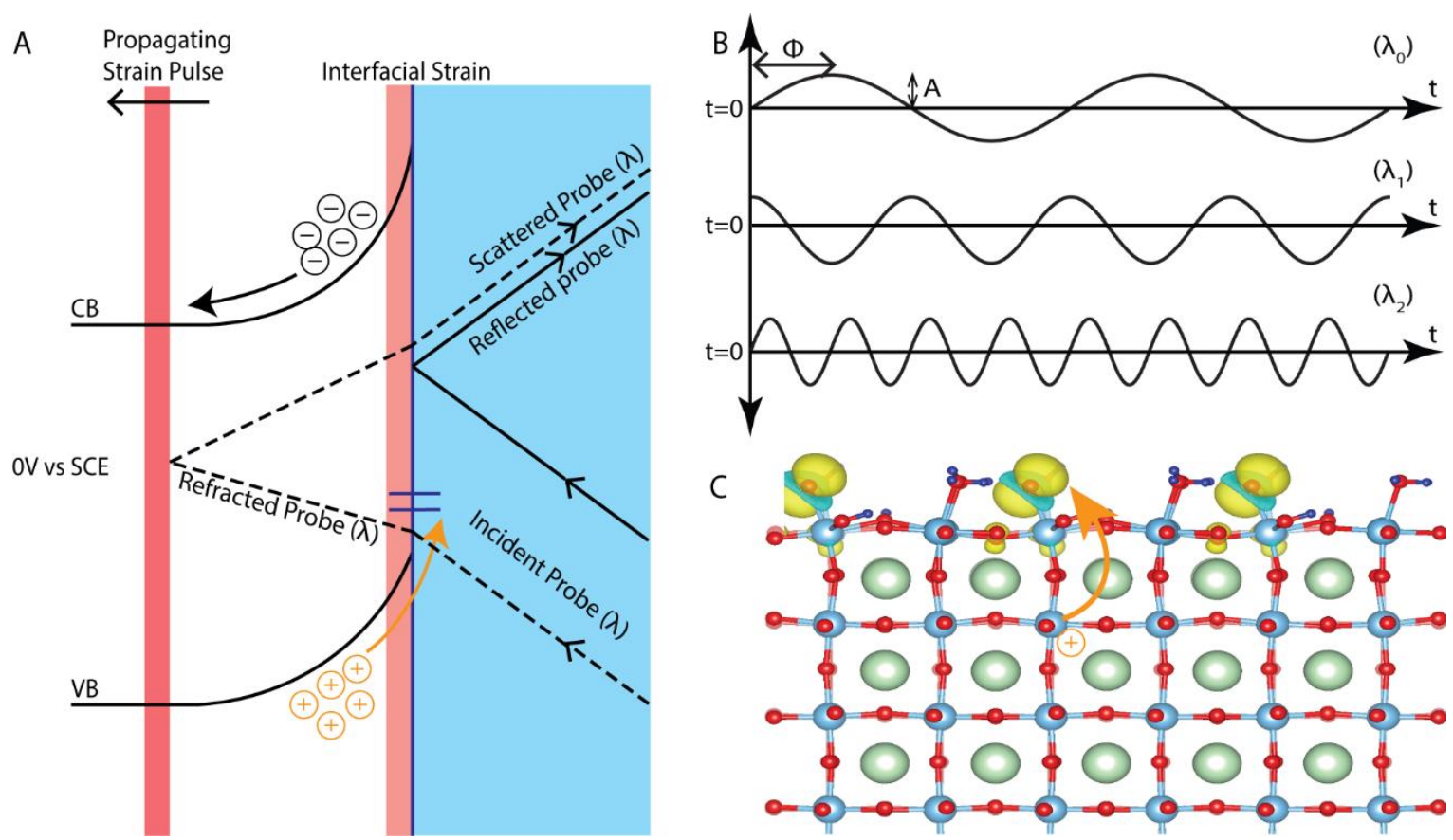

Figure 1: Overview of polaron-induced strain and detection of its propagating acoustic strain pulse. A) Band gap excitation generates carriers, which separate due to the space-charge layer. Holes trap at the surface (panel C) and cause an interfacial strain. Ultrafast generation of the strain launches a propagating strain pulse into the bulk, which we detect as an interference pattern between the surface reflected probe and acoustically scattered probe. We refer to this interference pattern as coherent acoustic waves (CAWs). B) As the pulse propagates into the bulk and the pathlength difference between the reflected and scattered probe increases, the CAWs oscillate in time with different wavelengths oscillating at different frequencies. These oscillations are characterized by their amplitude and phase. C) Small hole-polaron formation at the STO/water interface by hole-trapping.

Such considerations of interfacial strain fields have largely been based on the catalyst composition in the ground state and its stability against a steady-state potential. However, given that ${\mathrm{M}-\mathrm{OH}^{*}}^{*}$ itself induces lattice distortions, measured populations are meta-stable, and the closeness of unstable oxide phases to OER, determining the interfacial strain invoked by the M$\mathrm{OH}^{*}$ coverage itself would be important to understanding materials design paradigms for both 
higher activity and enhanced stability. Here, we employ a mature optical technique, ultrafast coherent acoustic interferometry, ${ }^{28-34}$ to detect the interfacial strain engendered by $\mathrm{Ti}-\mathrm{OH}^{*}$ coverage on $\mathrm{SrTiO}_{3}(0.1 \% \mathrm{Nb})$ during OER. As summarized in fig. 1A, the optical technique works by first generating a laser-triggered interfacial strain (usually associated with heat or an electron-phonon deformation potential) which results in a propagating acoustic strain pulse into the bulk. The optical probe pulse scatters off this propagating strain pulse with the phase matching conditions of stimulated Brillouin scattering (SBS). The scattered and surface reflected probe beams interfere to generate coherent acoustic waves (CAWs) in the data, defined by the oscillation frequency, amplitude, and phase response as a function of optical wavelength (fig. 1B). We newly apply this technique to OER, utilizing methods established previously to photo-drive OER at the $\mathrm{SrTiO}_{3} /$ aqueous interface ${ }^{35}$ by an ultrafast light pulse.

The coherent acoustic interferometry allows for completing the structural characterization of Ti-OH* on lightly-doped $\mathrm{SrTiO}_{3}(0.1 \% \mathrm{Nb})$, which arises from hole-trapping to the surface, as cartooned in fig. 1C. Previously, this intermediate had been characterized for the charge trapped through mid-gap electronic states probed by emission in the UV-visible regime ${ }^{8}$ and for the symmetry of the lattice distortions by the appearance of new normal modes ${ }^{12}$ (assigned to the terminal "oxyl" Ti-O*-) in the mid-infrared regime. Both the optical and mid-IR responses identified a 1.3 ps time scale for hole-trapping and $\mathrm{Ti}-\mathrm{OH}^{*}$ formation. The GHz-THz regime of acoustic waves now allows one to assign an interfacial strain to the $\mathrm{Ti}-\mathrm{OH}^{*}$ coverage achieved with a $0.04 \mathrm{~mJ} / \mathrm{cm}^{2}$ excitation associated with $\sim 2 \%$ of the surface sites. We apply a spectral analysis, further developed here, for detecting a combination of the spatial extent, magnitude, and generation time of the interfacial strain through especially the phase response of coherent acoustic waves. For $\mathrm{SrTiO}_{3}$, we identify the strain generation time $(1.31 \pm 0.02 \mathrm{ps})$ to occur simultaneously with Ti-OH* formation, and a tensile strain along the (100) crystal direction of $0.06 \%$ (upper limit $0.6 \%$ ). Below, this tensile strain, in which the $c$ axis expands with respect to the in-plane axes, is associated with lattice deformation mechanisms of how polarons create a continuum strain. Altogether, the methods developed herein are quite general and could be applied to differentiate photo-driven transition metal oxide catalysts by the interfacial strain coincident with $\mathrm{M}-\mathrm{OH}^{*}$, informing materials design paradigms for OER. 


\section{Experimental}

We acquired $0.08 \% \mathrm{Nb}$-doped $\mathrm{SrTiO}_{3}$ (STO) by weight (henceforth $0.08 \%$ is referred to as $0.1 \%$ ) single crystals of size $10 \times 10 \times 0.5 \mathrm{~mm}$ with crystallographic orientation (100) from MTI Corp. (Richmond, CA). Samples were used without any further chemical processing or annealing. All transient reflectance (TR) measurements were performed on STO samples with polished front sides $\left(\mathrm{R}_{\mathrm{a}}<8 \AA\right.$ ) and unpolished backsides. UV-VIS measurements for the visible STO absorption coefficient were performed on STO samples with both sides polished $\left(\mathrm{R}_{\mathrm{a}}<8 \AA\right.$ ). In addition, the solutions used were within the range of $\mathrm{pH} 7$ through $\mathrm{pH} 14$ with sodium hydroxide (Sigma Aldrich) and, where possible, the $\mathrm{Na}^{+}$concentration was kept at $100 \mathrm{mM}$ using sodium sulfate (Sigma Aldrich). The $\mathrm{pH}$ of the solutions was measured by a calibrated $\mathrm{pH}$ meter (Accumet $\mathrm{pH}$ electrode \#13-620-631 from Fisher Scientific).

All electrochemical measurements were performed with respect to a $\mathrm{Ag} / \mathrm{AgCl}(3 \mathrm{M} \mathrm{KCl})$ reference electrode (MF-2052; Basi) and controlled by a CHI650E Potentiostat (CH Instruments). For the working electrode, ohmic contact between the unpolished STO backside and a copper wire was established using a $\mathrm{Ga} / \mathrm{In}$ eutectic (Sigma-Aldrich). Exposed wire and the sample edges were covered with an insulating lacquer. A coiled Pt wire served as the counter electrode. Cyclic voltammetry was performed under light-on and light-off conditions with a scan rate of $100 \mathrm{mV} / \mathrm{s}$.

All TR measurements were performed in an in-situ electrochemical cell (fig. S1) under one of two conditions: OC and CC. For CC measurements, the sample was illuminated by the chopped $266 \mathrm{~nm}$ pump (light-on conditions) with a pulse energy of $15.7 \mathrm{~nJ}$ to give a pump fluence of 0.04 $\mathrm{mJ} / \mathrm{cm}^{2}$ (except the fluence dependent measurements), corresponding to carrier densities on the order of $10^{13} \mathrm{~cm}^{-2}$, while we acquired the photocurrent measured as a function of time (fig. S2) and while the potential of the STO photoelectrode was $0 \mathrm{~V}$ vs. SCE. For OC conditions, we used a pump energy of $785 \mathrm{~nJ}$ to give a pump fluence of $2.0 \mathrm{~mJ} / \mathrm{cm}^{2}$ (except the fluence dependent measurements) and all electrodes were disconnected from the potentiostat.

All measurements for phonon phase analysis were performed in a new Teflon in-situ electrochemical cell with a $1 \mathrm{~mm}$ UVFS window and $1 \mathrm{~mm}$ of solution between the sample and window to help minimize white light chirp. The raw data for these measurements is available in fig. S3. Measurements for the phonon amplitude correlation were performed with a similar in-situ sample cell ( $3 \mathrm{~mm} \mathrm{CaF}_{2}$ with $1 \mathrm{~cm}$ between the sample and window) and TR setup used previously. ${ }^{36}$ 
For the TR setup, the pump and probe beams were derived from a regeneratively amplified Ti:sapphire laser system (Coherent Legend; Coherent, Inc., Santa Clara, CA) producing pulses with a center wavelength of $800 \mathrm{~nm}$ and $\sim 200 \mathrm{fs}$ temporal width (fig. S4A) at a $1 \mathrm{kHz}$ repetition rate. The $266 \mathrm{~nm}$ pump was generated using a third harmonic generation setup (Eksma FK800-200-M) to generate $266 \mathrm{~nm}$ light with $\sim 400 \mathrm{fs}$ temporal width (fig. S4B) as the pump. The pump beam was modulated by a mechanical chopper (3501; Newport, Inc., Irvine, CA) at a frequency of $500 \mathrm{~Hz}$. The pump beam was incident normal with varying pulse energies and with a vertical and horizontal spot size of $200 \mu \mathrm{m}$ (FWHM), as measured by a knife edge. To maintain the fluence, we measured the pulse energy before each experiment using a pyroelectric energy sensor (919E-200U-8-25K) from Newport and took into account the window's and STO's reflectivity ( $20 \%$ loss).$^{36}$ For fluence calculation, we assumed the beam had a flat-top intensity profile with a diameter of the $200 \mu \mathrm{m}$.

The s-polarized white light continuum (WLC) probe was generated by focusing s-polarized $800 \mathrm{~nm}$ pulses into a rotationally translated $5 \mathrm{~mm}$ thick $\mathrm{CaF}_{2}$ crystal (Newlight Photonics) and delivered to the sample using reflective optics to minimize chirp. The leftover fundamental ( 800 $\mathrm{nm}$ ) was cut out using an $800 \mathrm{~nm}$ Notch Filter (Semrock) before the probe hits the sample and the broadened portion of the fundamental was cut out with a solution of two dyes (NIR783C \& NIR836C, QCR Solutions) in a cuvette after the sample but before the spectrometer to reduce grating ghosting artifacts. The incident angle of the probe on the sample cell was $45^{\circ}$ with a spectrally averaged spot size of $\sim 60 \mu \mathrm{m}$ when using a $650 \mathrm{~nm}$ short pass filter (FES0650, Thorlabs). Note that the focus was not spectrally uniform and that the blue end of the spectrum did not focus as tightly, which we did not further characterize.

After the sample, the reflected probe beam was focused into an imaging spectrograph (Isoplane) and CCD Imaging Camera (PIXIS) setup from Princeton Instruments. In addition, $<10 \%$ of the probe was split before the sample for referencing. Referencing was performed with an un-matched CMOS UltraFast Systems spectrometer using the smart referencing algorithm ${ }^{37}$ with a 64 compressed pixel array ${ }^{38}$ to achieve a differential probe stability of approximately 2 mOD per pulse pair. The detector outputs were interfaced with a personal computer, which provided automated control over a double-pass 4 ns optical pump-probe delay stage (MTM250CC1; Newport/MKS). 
Finally, under light on CC conditions, the sample undergoes surface degradation (fig. S5), which affects the photocurrent, TR signal, and sample reflectivity. Surface degradation does not occur under the OC conditions tested in this work. To minimize the signal artifacts associated with this degradation, we mounted the sample on a 3-axis stage controlled by programmable actuators (Z 825 B; Thorlabs, Inc.) and controllers (TDC001; Thorlabs, Inc.). We raster scanned the sample at a controllable speed of $7 \mu \mathrm{m}$ and minimized the signal artifacts associated with this degradation (fig. S5B). More detail on surface degradation is given in section S4.

\section{Results}

We performed picosecond white light transient reflectance (TR) spectroscopy on $0.1 \%$ $\mathrm{Nb}: \mathrm{SrTiO}_{3}$ (STO) under OC and CC conditions. For CC conditions at $0 \mathrm{~V}$ vs. SCE, bandgap excitation with a 400 fs $266 \mathrm{~nm}$ pump generated a photocurrent with > 70\% quantum efficiency (fig. S2B) and drove the oxygen evolution reaction (OER) with $\sim 100 \%$ Faradaic efficiency. ${ }^{35}$ Both OC and CC TR spectra contained prominent $\sim 50-100 \mathrm{GHz}$ oscillations that we attribute to CAWs. These oscillations are shown in fig. 2A. They are an additive contribution to the optical transitions (fig. $2 \mathrm{~A}$, phonon removed) which we have studied in a previous publication. ${ }^{36}$ In the next three sections, we will extract the frequency (3.1), amplitude (3.2), and phase (3.3) of these oscillations and use them to link the CAWs in CC to interfacial strain generated by hole-trapping to create Ti$\mathrm{OH}^{*}$. The OC condition will be used as a foil, in which the CAWs derive instead from high photocarrier populations. Furthermore, in the phase section (3.3), we develop a mathematical model of the CAWs as a function of optical wavelength and fit it to our experimental CAW phase to extract a $~ 1.3$ ps strain formation time in CC and directly link an interfacial and uniaxial (100) tensile strain to polaron formation. This quantitative model will also allow us to estimate the strain's

magnitude and a lower bound to the strain's spatial extent, using both the CAW phase and amplitude. 


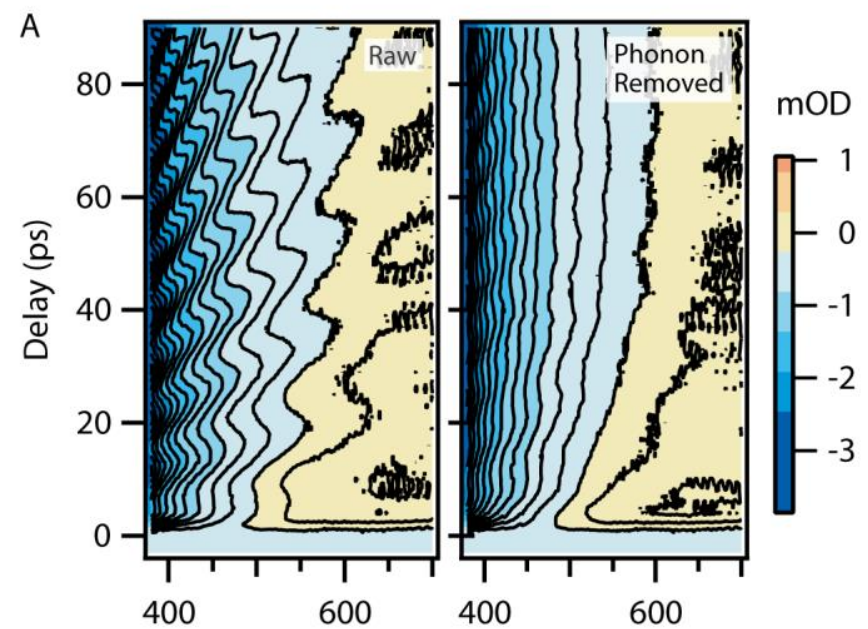

Wavelength $(\mathrm{nm})$
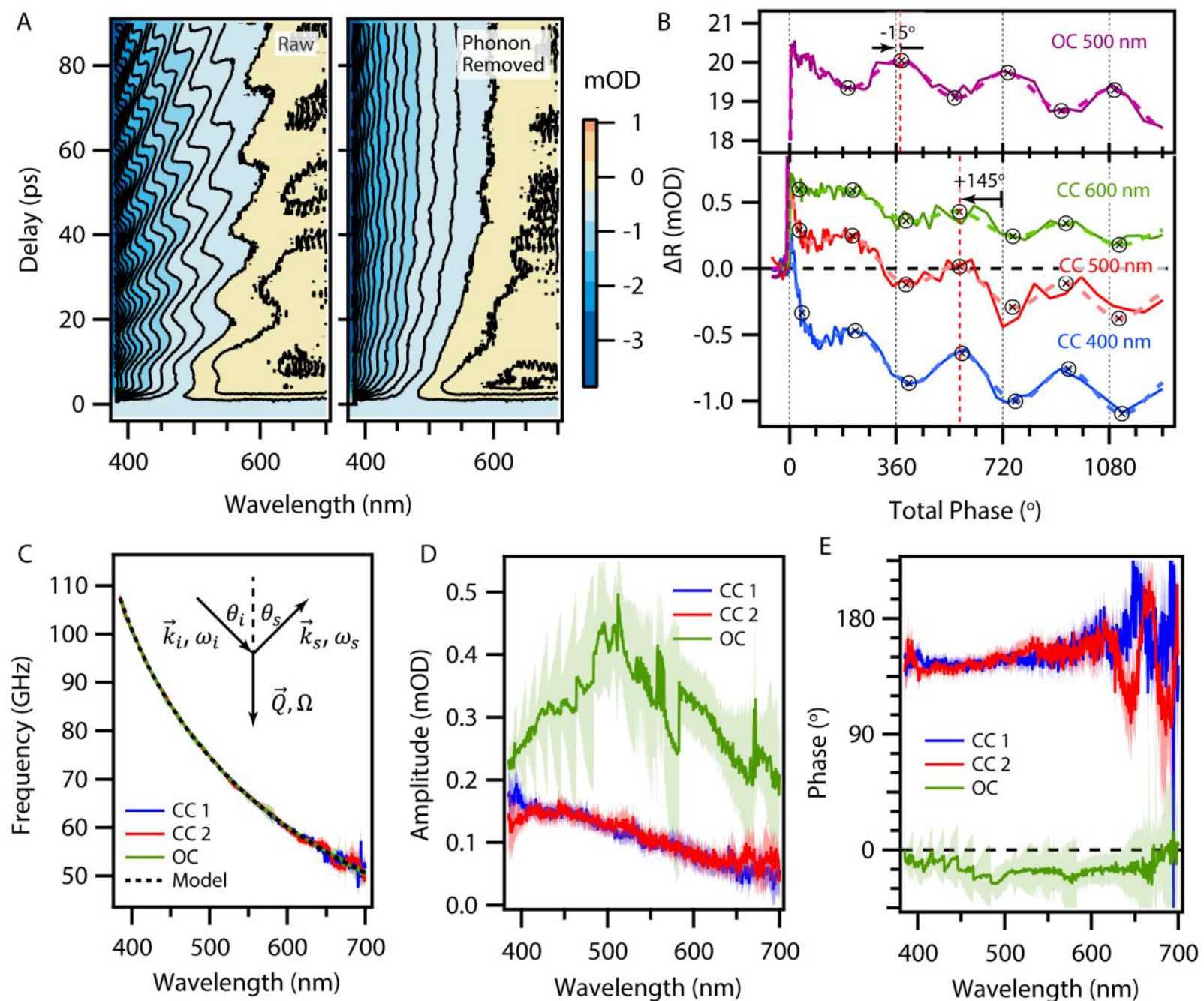

Figure 2: A) Example TR data with (left) and without (right) the CAW contribution. B) Equation 1 fit traces for select wavelengths for OC (top) and CC 2 (bottom) conditions. The xaxis is given in terms total phase, $\phi=\Omega(\lambda)_{\mathrm{STO}} t$, to emphasize the phase shifts in the oscillations. The vertical red lines indicate the average phase shift, $\phi_{\mathrm{STO}}$. The crossed circles

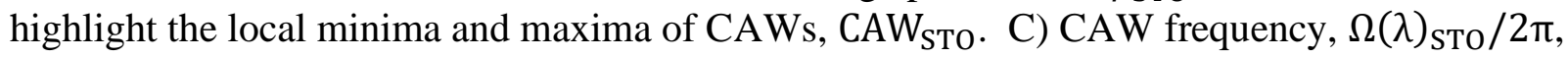
as a function of probe wavelength as extracted by the fit via eq. 1 . The inset shows the wavevectors for the SBS phase matching conditions. $\vec{k}, \omega$, and $\theta$ represent the optical wavevector, frequency, and angle while the subscripts i and s represent incident and Stokes, respectively. $\vec{Q}$ and $\Omega$ are the longitudinal acoustic phonon wavevector and frequency. D) The CAW amplitude, $\mathrm{A}_{\mathrm{CAW}, \mathrm{STO}}$, as a function of wavelength for OC and two CC data sets as extracted by the fit via eq. 1 . E) The CAW phase, $\phi_{\text {STO }}$, as a function of wavelength for OC and two CC data sets as extracted by the fit via eq. 1. For all panels, OC conditions were acquired at $\mathrm{pH} 13$ with a pump fluence of $2.0 \mathrm{~mJ} / \mathrm{cm}^{2}$ and $\mathrm{CC}$ conditions were acquired at $\mathrm{pH} 13$ with a pump fluence of $0.04 \mathrm{~mJ} / \mathrm{cm}^{2}$. The shaded regions of C, D, and E represent the standard error bounds from the least squares fit. For all panels, the raw TR data used to generate the CAW data in this figure is shown in fig. S3. 


\subsection{CAWs Frequency:}

We extracted the wavelength-dependent oscillation frequency, amplitude, and phase by fitting the TR kinetic traces for each wavelength to a sum of two growth exponentials, a decay exponential, and oscillatory components that models CAWs in STO and water (fig. 2B):

$$
\begin{array}{cc}
\Delta O D=G_{\text {fast }}+G_{\text {slow }}+D_{1}+C A W_{\text {STO }}+C A W_{H 2 O}, & 1 \mathrm{a} \\
G_{i}=-A_{G, i}\left(1-\exp \left(-t / \tau_{G, i}\right)\right) & 1 \mathrm{~b} \\
D_{i}=A_{D, i} \exp \left(-t / \tau_{D, i}\right) & 1 \mathrm{c} \\
C A W_{i}=A_{C A W, i} \exp \left(-t / \tau_{C A W, i}\right) \cos \left(\Omega_{i} t+\phi_{i}\right) & 1 \mathrm{~d}
\end{array}
$$

The two growth exponentials in eq. 1 represent the growth of an emissive signal associated with hole trapping and the decay exponential is associated with the decay of the valance band hole population (described below). ${ }^{8,9,12,36,39}$ For hole trapping, we found that the average fast growth timescale was $1.2 \pm 0.3$ ps (fig. S6). The two CAW terms are associated with acoustic pulse propagation in STO and in the aqueous electrolyte (fig. S7). The $\sim 5-10 \mathrm{GHz}$ signal contribution from the electrolyte is described in section S5. The wavelength dependent fit results for STO CAWs frequency, amplitude, and phase are summarized in fig. 2C-E, respectively.

We found that the oscillation frequency as a function of probe wavelength matches the stimulated Brillouin scattering (SBS) phase matching condition almost exactly, as shown in fig. 2C, with a small error related to spectrometer calibration (fig. S8). The SBS phase matching condition is given by, $28,34,40,41$

$$
\frac{\Omega}{2 \pi}=\frac{2 v n}{\lambda} \cos \theta_{i}
$$

where $\lambda$ is the probe vacuum wavelength, $n(\lambda)$ is the real part of the index of refraction in $0.1 \%$ $\mathrm{Nb}: \mathrm{STO}$ (fig. S9), $v$ is the acoustic velocity in STO (calculated in Table 1 ), ${ }^{42}$ and $\theta_{i}(\lambda)$ is the incidence angle of the probe in STO as defined in the inset of fig. 2C. In addition, the CAWs in water also closely follows this relation (fig. S7), with the corresponding refractive index, acoustic velocity, and incidence angle. These close agreements indicate that the oscillatory signals are CAWs as detected by picosecond interferometry (as opposed to a resonantly enhanced mechanism, ${ }^{43,44}$ which does not follow the SBS phase matching conditions). For STO, the physical picture that follows is cartooned in fig. 1: the interference of the surface reflected probe and transmitted probe scattering from an acoustic strain pulse that propagates into the bulk of the 
sample. ${ }^{28,30}$ This acoustic strain pulse is a direct result of ultrafast strain generation just below the STO surface, which describes uniaxial expansion (tensile strain) or contraction (compressive strain) of the STO lattice in a direction normal (100) to the surface (001).

\subsection{CAWs Amplitude}

To gain more insight into the source of the interfacial strain, we correlated the CAW amplitude against the amplitudes of the fast emissive component and absorptive component while varying electrolyte $\mathrm{pH}$ for $\mathrm{CC}$ conditions and pump fluence for $\mathrm{OC}$ and $\mathrm{CC}$ conditions, as summarized in fig. 3. These amplitudes were extracted by performing a spectrally constrained singular value decomposition (SVD) analysis, which yields a single pair of kinetic traces for the absorptive and emissive component (fig. S10). We fit the resulting kinetic traces to the exponential parts $\left(G_{\text {fast }}, G_{\text {slow }}\right.$, and $\left.D_{1}\right)$ of eq. 1a to extract a condition-dependent absorptive amplitude which arises within the excitation pulse width (<400 fs) and a condition-dependent emissive amplitude which formed with a $\sim 2$ ps time constant. The advantage of this method is that it allows us to extract condition-dependent kinetic parameters rapidly and robustly from large data sets without the added complexity of fitting multiple probe wavelengths. We emphasize that the $\sim 2$ ps emissive component and <400 fs absorptive component is analogous to the amplitudes derived from the emissive rise and absorptive decay exponentials described in eq. 1. More detail is provided in the SI (section S9) and in previous publications. ${ }^{36}$

Before proceeding, we will introduce the relevant differences between $\mathrm{OC}$ and $\mathrm{CC}$ conditions in our STO system, which are summarized in fig. S11. Briefly, under OC conditions with our range of pump fluence, interfacial dynamics are dominated by photocarrier recombination because the built-in potential at the STO/water interface flattens. ${ }^{9}$ Similarly, the OC TR spectra are dominated by a decaying absorptive component that forms within the pump pulse duration, which we therefore associate with the photocarrier populations. In contrast, under CC conditions the built-in potential is maintained, causing the photocarriers to separate and to generate a photocurrent (fig. S2).${ }^{9}$ In this process, the valance band holes are driven to the surface and trap as

surface terminal and bridged oxyls, with the associated hole-polaron mid-gap states (fig. S11). ${ }^{8}$ We have previously assigned the $\sim 2$ ps emissive component to the transition between the conduction band and the hole-trapped states to this $\mathrm{Ti}^{-\mathrm{OH}^{*}}$ population. $^{8}$ 

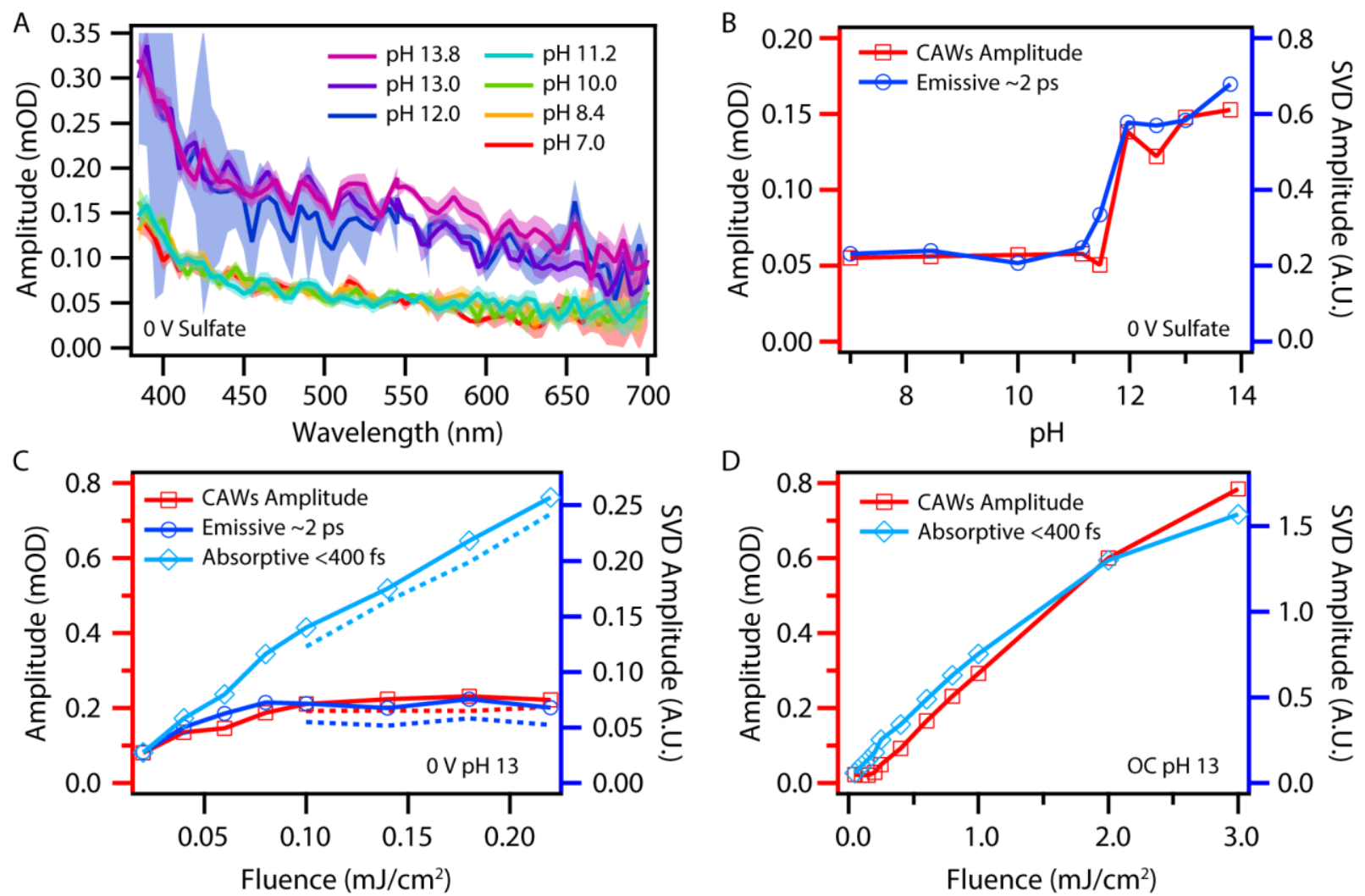

Figure 3: Correlating CC CAWs amplitude to the $\sim 2$ ps emissive amplitude (polaron formation) and OC CAWs to the <400 fs absorptive amplitude (carrier population). A) The CAW amplitude spectrum, as extracted by eq. 1 , for $\mathrm{CC}$ conditions for various pHs. B) The average CAW amplitude (red squares, left axis) for the data set in A correlated with the $\sim 2 \mathrm{ps}$ emissive amplitude (blue circles, right axis). C) Fluence dependance for $\mathrm{CC}$ in $\mathrm{pH} 13$. Here, the CAW amplitude (red squares, left axis) correlates with the $\sim 2$ ps emissive amplitude (dark blue circles, right axis), but not with the $<400 \mathrm{fs}$ absorptive amplitude (light blue diamonds, right axis). To show that the effects of surface degradation do not influence the correlation, the solid lines were acquired with a scan speed of $7 \mu \mathrm{m} / \mathrm{s}$ while the dashed lines were acquired at $28 \mu \mathrm{m} / \mathrm{s}$ (see section $\mathrm{S} 4$ for more detail). D) Fluence dependance for $\mathrm{OC}$ in $\mathrm{pH} 13$ showing that the OC CAW amplitude (red squares, left axis) correlates with the $<400$ fs absorptive amplitude (light blue diamonds, right axis). For all panels, the $\sim 2$ ps emissive amplitude and $<400$ fs absorptive amplitude were extracted by fitting kinetic components from constrained SVD analysis (see section S9).

Figure $3 \mathrm{~A}$ and $2 \mathrm{~B}$ show the most prominent features for varying $\mathrm{pH}$ in $\mathrm{CC}$, where there is a stark difference in the CAWs amplitude spectra between neutral and basic pH. In fig. 3A, we show that the shape of the spectra within this data sets is largely the same, indicating that the average CAW amplitude within a single data set (from 400 to $600 \mathrm{~nm}$ ) can be reliably used to compare against the emissive and absorptive amplitudes. In fig. 3B, we show that the average CAW amplitude (red squares) tracks the sigmoidal shape of the $\sim 2$ ps emissive amplitude (blue 
circles) almost exactly. This sigmoidal shape results from modulating the number of available surface hole trap sites through the surface acid-base equilibrium modulated by $\mathrm{pH}$ of the electrolyte. The shape is consistent with an adsorption reaction isotherm at a surface or a Langmuir isotherm, as detailed elsewhere. ${ }^{36}$

Further, when varying pump fluence, the correlation between the CAW amplitude and the emissive amplitude assigned to $\mathrm{Ti}^{-} \mathrm{OH}^{*}$ is present only under $\mathrm{CC}$ conditions and not under $\mathrm{OC}$ conditions. As shown in fig. 3C, the CC CAW amplitude (red squares) and emissive amplitude (dark blue circles) both exhibit a saturation behavior as the pump fluence is increased, while the absorptive amplitude (light blue diamonds) grows linearly with fluence. In contrast, the OC CAW amplitude (fig. 3D, red squares) closely follows the nearly linear increase of the absorptive amplitude (light blue diamonds) and does not exhibit any saturation behavior for the wide range of fluences measured. Further, when normalized for fluence, the CAWs signal is approximately 10 times stronger in $\mathrm{CC}$ as compared to $\mathrm{OC}$ within the $\mathrm{CC}$ linear regime. This can be seen by comparing the CC $0.2 \mathrm{mOD}$ CAW amplitude at a fluence of $0.08 \mathrm{~mJ} / \mathrm{cm}^{2}$ in fig. $3 \mathrm{C}$ with the $\mathrm{OC}$ 0.2 mOD CAW amplitude in fig. 3D, which occurs at a fluence of $0.8 \mathrm{~mJ} / \mathrm{cm}^{2}$. That the CAW amplitude correlates with the $\mathrm{pH}$ dependence and saturation behavior of the $\sim 2$ ps emissive amplitude strongly attributes the $\mathrm{CC}$ generation to hole-polaron or $\mathrm{Ti}-\mathrm{OH}^{*}$ formation. On the other hand, the linear correlation of the CAW amplitude with fluence that patterns the absorptive amplitude, along with its less efficient excitation, attributes the strain formation in OC to high photo-carrier populations. 


\subsection{CAWs Phase Model}

A

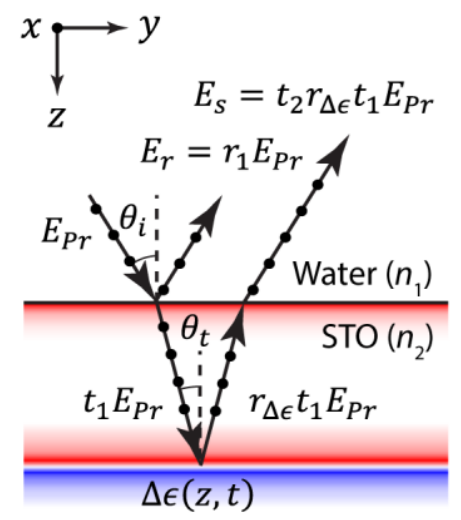

B

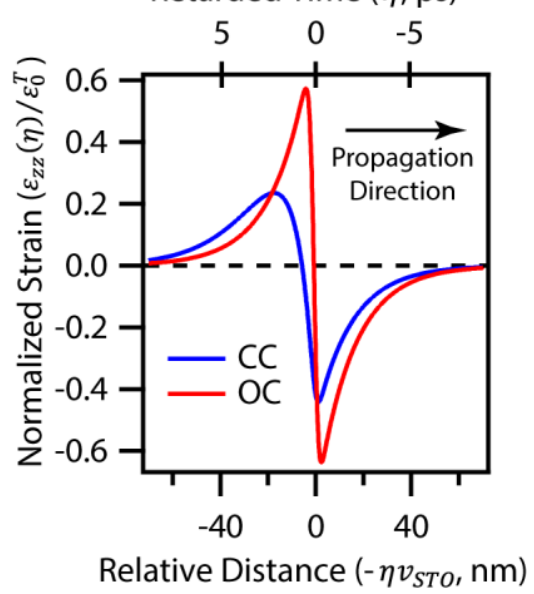

Figure 4: A) The reflectance, E/M part of the CAWs model: a diagram defining the coordinate system and symbols used to model the CAWs interference signal and probe scattering from a change in permittivity, $\Delta \epsilon(z, t)$, caused by the acoustic strain pulse, $\varepsilon_{z z}$. B) The acoustic strain part of the model: the acoustic strain pulse, $\varepsilon_{z z}(\eta)$ in eq. S33, normalized to the transformation strain amplitude, $\varepsilon_{0}^{T}$, that generates it. For comparison, both CC and OC conditions were chosen to have a spatial extent of $15 \mathrm{~nm}$ and $\mathrm{CC}$ had a formation time of $1.2 \mathrm{ps}$, while OC had a formation time of $0.1 \mathrm{ps}$.

We now turn to the model of the CAWs amplitude and phase spectra in terms of the sign, magnitude, spatial extent, and formation time of the interfacial strain that generates the propagating strain pulse. We then fit the model to our experimental CC CAWs amplitude and phase spectra, which allows us to obtain a uniaxial (001) tensile strain that leads to $c>a$, extract a $\sim 1.3$ ps interfacial strain formation time, and estimate an interfacial strain magnitude with a lower bound spatial extent. This model will extend the phenomenological model initially developed by Thomsen, et al. ${ }^{28}$ in the acoustic interferometry regime ${ }^{45}$ by including a strain formation time and broadband response. In addition, we will primarily focus on the CAWs phase spectrum because we found that the CAWs amplitude spectrum was susceptible to systematic error related to our white light continuum mode quality. Note that a similar formation time vs. phase analysis has also been performed by Babilotte, et al. ${ }^{46}$

Below, we summarize the key features of the model, which are plotted in fig. 4. The theory of picosecond acoustic interferometry is typically partitioned into the detection and generation mechanism. In analogy, we have also partitioned fig. 4 into the reflectance, E/M part (A) and acoustic strain part (B), where fig. 4A shows the generation of the CAWs interference pattern in 
terms of the probe's electric field and fig. 4B shows the propagating acoustic pulse that scatters the probe. More detail is provided in the SI, along with the key assumptions used in the model.

For the reflectance, E/M part of the model, the signal that we detect is the interference between the surface reflected probe, $E_{r}$, and acoustic scattered probe, $E_{S}$, as summarized in fig. 4A. This interference, $\left|E_{r}+E_{s}\right|^{2}$, leads to the CAWs oscillations seen in our data and those sketched in fig. 1B as the acoustic pulse propagates away from the surface. For small signals, the interference signal in the absorption convention ${ }^{8}$ (an increase in reflectivity corresponds to a negative $\triangle O D$ value) is:

$$
\begin{gathered}
\Delta O D \approx \frac{2\left|r_{1}(\lambda) t_{1}(\lambda) t_{2}(\lambda) r_{\Delta \epsilon}(\lambda, t)\right|}{\ln (10)\left|r_{1}(\lambda)\right|^{2}} \cdot \cos \left(\phi_{\text {total }}(\lambda, t)\right) \\
\phi_{\text {total }}(\lambda, t)=180^{\circ}-\phi_{r 1}(\lambda)+\phi_{t 1}(\lambda)+\phi_{t 2}(\lambda)+\phi_{r \Delta \epsilon}(\lambda, t)
\end{gathered}
$$

Note that, in analogy to eq. 1, we have partitioned each complex coefficient in terms of its magnitude and phase and added the negative sign for the absorption convention into the phase as a factor of $180^{\circ}$. Here, $r_{1}(\lambda), t_{1}(\lambda)$, and $t_{2}(\lambda)$ are the complex Fresnel coefficients for probe reflection at the surface, transmission into STO, and transmission out of STO, respectively. These coefficients are calculated from the complex index of refraction $\tilde{n}(\lambda)=n(\lambda)+i \kappa(\lambda)$ for water $^{47,48}$ and $0.1 \% \mathrm{Nb}: \mathrm{STO}$ (fig. S9A). ${ }^{9}$

The remaining term, $r_{\Delta \epsilon}(\lambda, t)$, is the probe reflection coefficient from the propagating acoustic strain pulse. In the limit that the acoustic pulse has completely left the surface, the reflection coefficient can be written in terms of the acoustic frequency domain spectrum, $\tilde{\varepsilon}_{z z}(\Omega(\lambda))$. Here, $\tilde{\varepsilon}_{z z}(\Omega(\lambda))$ is the Fourier-transform of the time-domain acoustic strain pulse, $\varepsilon_{z z}(\eta)$ with $\eta=t-z / v_{\text {STO }}$ (shown in fig. $4 \mathrm{~B}$ ), in the absence of any acoustic damping mechanism. With these approximations, and for s-polarized light ( $E$-field in $x$ ) propagating in the $y z$-plane with an incidence angle of $\theta_{t}$, the $1^{\text {st }}$ order scattering coefficient is: ${ }^{49}$

$$
r_{\Delta \epsilon}(\lambda, t)=\frac{i \pi v_{\text {STO }}}{\lambda \tilde{n}(\lambda) \cos \left(\theta_{t}\right)} \frac{\Delta \epsilon_{x x}(\lambda)}{\Delta \varepsilon_{z Z}} \tilde{\varepsilon}_{z z}(\Omega(\lambda)) e^{i \Omega(\lambda) t},
$$

where, assuming an isotropic crystal and uniaxial strain, $\Delta \epsilon_{x x}(\lambda) / \Delta \tilde{\varepsilon}_{z z}(\lambda)=$ $\epsilon_{x x}(\lambda) p_{x x z z}(\lambda) \epsilon_{x x}(\lambda)$ is elasto-optic contribution and $p_{x x z z}(\lambda)$ is the relevant elasto-optic tensor element.

For the acoustic strain part of the model, the second half of the theory is the strain generation mechanism, which models the interfacial strain and its resulting acoustic pulse, $\varepsilon_{z z}(\eta)$ 
(fig. 4B). We follow the setup of the problem given by Bushnell and McCloskey. ${ }^{50}$ Briefly, the model is composed of two linear-elastic half-spaces which represent the water and STO interface. The main input parameter for this model is the isotropic transformation strain, or eigenstrain, ${ }^{51} \varepsilon_{z z}^{T}$ in eq. 5 below. The transformation strain describes the spatial and temporal dependance of how the lattice transforms (expands or contracts) under conditions of stress-free volume expansion, in analogy to thermal expansion or chemical expansivity. ${ }^{52}$ Because our pump diameter (>200 $\left.\mu \mathrm{m}\right)$ is much larger than the acoustic pulse propagation distance $(<8 \mu \mathrm{m})$ that we analyze, we assume translational symmetry in the in-plane coordinates $(x$ and $y)$ and approximate our strain as uniaxial, which reduces the problem to one dimension along $z$.

We model the transformation strain as:

$$
\begin{array}{cc}
\varepsilon_{z z}^{T}(z, t)= & \varepsilon_{0}^{T} S(z / \xi)\left(G\left(t / t_{0}\right) * P(t / \sigma) / \sigma\right), \\
S(x)=\theta(x) e^{-x}, & 5 \mathrm{a} \\
G(x)=\int_{-\infty}^{x} g(y) d y=\theta(x)\left(1-e^{-x}\right), & 5 \mathrm{c} \\
P(x)=(2 \pi)^{-1 / 2} e^{-\frac{x^{2}}{2}}, & 5 \mathrm{~d}
\end{array}
$$

where * represents convolution over $t, \varepsilon_{0}^{T}$ is the strain magnitude, $\xi$ is the spatial extent, $t_{0}$ is the strain formation time, $\sigma$ is the pump intensity duration, and $\theta(x)$ is the Heaviside step function. Equation $5 \mathrm{~b}$ represents the spatial dependance of the transformation strain along the $z$-axis, which we assumed to be an exponential decay. Equation 5c represents the transformation strain's temporal dependence, which we set to be a $1^{\text {st }}$ order exponential growth process. Note that this equation is explicitly written as an integral because the solution for the strain, eq. 6, will eventually be written in terms of the growth rate, $g(y)$. Finally, we include finite pump-pulse effects by convolving eq. $5 \mathrm{c}$ with the pump-pulse intensity profile in eq. $5 \mathrm{~d}$. We took the FWHM pulse duration to be $0.4 \mathrm{ps}$ (fig. S4).

Table 1: Constants, at room temperature, used for the CAWs model.

Quantity Value




\begin{tabular}{|c|c|}
\hline Lattice Constant (STO) ${ }^{42}$ & $0.3904 \mathrm{~nm}$ \\
\hline Density $^{\alpha, 53}$ & $\begin{array}{l}\rho_{\text {STO }}=5.12 \mathrm{~g} / \mathrm{cm}^{3} \\
\rho_{\text {H2O }}=0.998 \mathrm{~g} / \mathrm{cm}^{3}\end{array}$ \\
\hline Acoustic Velocity ${ }^{\beta, 54}$ & $\begin{array}{l}v_{\text {SтO }}=7.86 \mathrm{~nm} / \mathrm{ps} \\
v_{\text {H2O }}=1.49 \mathrm{~nm} / \mathrm{ps}\end{array}$ \\
\hline Bulk Modulus ${ }^{42}$ & $B_{\text {STO }}=170.57 \mathrm{GPa}$ \\
\hline Shear Modulus ${ }^{42}$ & $G_{\mathrm{STO}}=108.46 \mathrm{GPa}$ \\
\hline Poisson's Ratio (STO) ${ }^{42}$ & $v=0.277$ \\
\hline Thermal Expansion Coeff. (STO) $)^{55}$ & $\alpha_{V}=3.23 \times 10^{-5} \mathrm{~K}^{-1}$ \\
\hline Volumetric Heat Capacity (STO) ${ }^{55}$ & $C_{p}=2.730 \mathrm{~J} /\left(\mathrm{cm}^{3} \mathrm{~K}\right)$ \\
\hline Elasto-Optic Coefficient (STO $)^{56}$ & $p_{1122}=0.095$ at $633 \mathrm{~nm}$ \\
\hline Acoustic Reflection Coefficient ${ }^{\gamma}$ & $R=0.928$ \\
\hline
\end{tabular}

${ }^{\alpha}$ Calculated from the lattice constant and molar mass of STO.

${ }^{\beta}$ Compressive acoustic velocity calculated from the elastic moduli and density of STO.

${ }^{\gamma}$ Calculated $^{50}$ from the densities and acoustic velocities of STO and water.

Given the transformation strain in eq. 5, the acoustic frequency domain solution for the propagating strain pulse is given by:

$$
\tilde{\varepsilon}_{z Z}(\Omega(\lambda))=-\frac{3 B_{\mathrm{STO}} \varepsilon_{0}^{T} \xi}{2 v_{\text {STO }}^{3} \rho_{\text {STO }}} \tilde{T}\left(\frac{\xi \Omega(\lambda)}{v_{\text {STO }}}\right) \tilde{g}\left(t_{0} \Omega(\lambda)\right) \tilde{P}(\sigma \Omega(\lambda)),
$$

where the constants are summarized in Table 1 and where the functional forms of the Fourier transforms, $\tilde{T}(\omega), \tilde{g}(\omega)$, and $\tilde{P}(\omega)$, are given in eq. S34 and the phases of $\tilde{T}\left(\xi \Omega(\lambda) / v_{\text {STO }}\right)$ for various values of $\xi$ and $\tilde{g}\left(t_{0} \Omega(\lambda)\right)$ for various values of $t_{0}$ are plotted in fig. S13. $\tilde{T}\left(\xi \Omega(\lambda) / v_{\text {STO }}\right)$ is defined by the Fourier transform of $T\left(\eta v_{S T O} / \xi\right)=h(\eta) S\left(|\eta| v_{\text {STO }} / \xi\right)$, where $\eta=t-z / v_{\text {STO }}$ and where we have dropped the pre-factors associated with Fourier scaling for clarity. Here, $h(\eta)$ is a step function such that $h(\eta<0)=1$ and $h(\eta>0)=-R$ and where $R$ is the STO/water acoustic amplitude reflection coefficient. $S\left(|\eta| v_{S T O} / \xi\right)$ derives from the exponentially decaying spatial dependence of the interfacial strain (eq. 5b) and its reflection from the interface as it gets generated, while $h(\eta)$ accounts for the sign flip of the strain during reflection and its partial transmission into water. Together, $T\left(\eta v_{\text {STO }} / \xi\right)$ spatiotemporally describes the acoustic pulse propagating into the bulk that results from an instantaneously generated interfacial strain $\left(t_{0}=0\right)$. We therefore call $T\left(\eta v_{\text {STO }} / \xi\right)$ and its Fourier transform, $\tilde{T}\left(\xi \Omega(\lambda) / v_{\text {STO }}\right)$, the spatial part of the strain. However, fig. 4B graphs the full $\varepsilon_{z z}(\eta)$, which represents the acoustic propagating pulse in fig. 1A for a given strain growth rate and spatial extent. Since the strain is instantaneously 
generated in the OC condition (as described below), $T\left(\eta v_{\text {STO }} / \xi\right)$ directly leads to the OC pulse shape shown in fig. 4B for a given spatial extent, $\xi$, of the interfacial strain. The second term in eq. $6, \tilde{g}\left(t_{0} \Omega(\lambda)\right)$, is the Fourier transform of $g\left(\eta / t_{0}\right)$, the transformation strain's growth rate, which we will call the temporal function. In the spatiotemporal domain, eq. S33, a finite growth rate smears the spatial function, $T\left(\eta v_{\text {STO }} / \xi\right)$, (as a convolution) to give a pulse that resembles the $\mathrm{CC}$ pulse shape in fig. 4B. Finally, the third term is the normalized pump intensity profile and is a symmetric function that does not contribute a net phase to the total CAW phase (eq. 3b.)

Using the acoustic frequency domain solution for the propagating strain pulse, $\tilde{\varepsilon}_{z z}(\Omega(\lambda))$, eq. 6 , Figure $5 \mathrm{~A}$ shows how the model's average $(400-700 \mathrm{~nm})$ total phase $\left(\phi_{\text {total }}(\lambda, t)\right.$, eq. $\left.3 \mathrm{~b}\right)$ varies as a function of formation time for different spatial extents. In this figure, there are two important implications on the scope of conclusions which we can draw from fitting the model to our phase data. The first and more important implication is that the total CAWs phase is sensitive to small spatial extents and fast formation times but is insensitive to large spatial extents and slow formation times. This conclusion arises because the phases of the spatial and temporal functions that determine $\phi_{r \Delta \epsilon}(\lambda, t)$ in eq. $3 b$ have asymptotic relationships with spatial extent $(\xi)$ and formation time $\left(t_{0}\right)$. This is most clearly shown in fig. 5A: as the spatial extent approaches and exceeds $5 \mathrm{~nm}$, the curves converge to the $15 \mathrm{~nm}$ curve, thereby decreasing the model's phase sensitivity to large spatial extents. Similarly, the slope magnitude of any individual curve decreases with larger formation time, indicating that the model becomes insensitive to formation times greater than $\sim 15 \mathrm{ps}$. Therefore, when parameterized for STO, our model can reliably extract spatial extents of less than $\sim 5 \mathrm{~nm}$ and formation times of less than $\sim 15 \mathrm{ps}$. 

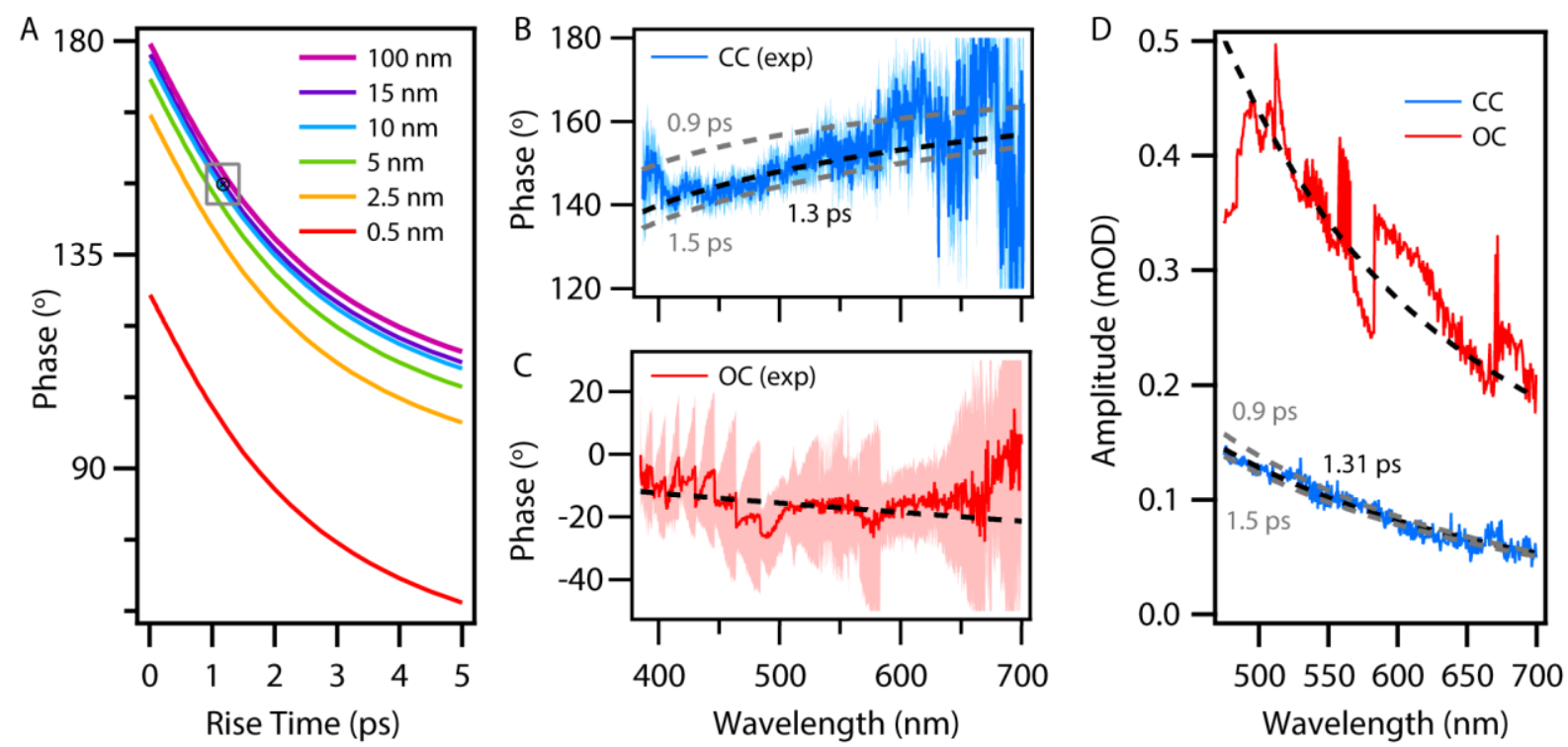

Figure 5: A) The dependance of the average phase (averaged from 375 to $700 \mathrm{~nm}$ ) on the strain's formation time and spatial extent. The slope of each curve indicates the CAWs model's sensitivity to the strain's growth rate. The box shows the average phase of the 1.31 ps fit in panel $\mathrm{B}$ and the standard deviation of its residuals, $150 \pm 4^{\circ}$, and the formation time constrained to be within the emissive growth rate, $\left\langle\tau_{G, \text { fast }}\right\rangle_{\lambda}=1.2 \pm 0.3$ ps as fit by eq. 1 (fig. S6). B) The CAWs model best fit (black dashed line, $1.31 \pm 0.03 \mathrm{ps}, 100 \pm 195 \mathrm{~nm}$ ) for the CC CAWs phase spectrum. To give a sense of our model's error bounds, we also show formation times of $0.9 \mathrm{ps}$ and $1.5 \mathrm{ps}$, both at $100 \mathrm{~nm}$, in panel B as grey dashed lines. These bounds correspond to the upper left and lower right corner of the box in panel A. C) The CAWs model best fit (black dashed line) to the OC CAWs phase spectrum. D) OC (red) and CC (blue) best fit curves for the CAWs amplitude spectrum with spatial extent and formation time constrained to the best fits in $\mathrm{B}$ and $\mathrm{C}$. In analogy to panel $\mathrm{B}$, for $\mathrm{CC}$ we show the effect of formation time on the CAWs amplitude while keeping the strain amplitude at $0.02 \%$ and spatial extent at $100 \mathrm{~nm}$. For all panels, the CC data is an average of the fig. 2 results for CC 1 and CC 2. In addition, the X-axis range shows the model fit range of the data and shaded regions show the standard error weights used to fit the phase.

The second implication is that the spatial and temporal phase contributions are additive toward the total phase. This can be seen in fig. $5 \mathrm{~A}$, where the phase vs. formation time curves have the same shape but differ by a vertical offset, which is the phase contribution from the spatial extent. The implication is that for a single probe wavelength, one would not be able to simultaneously extract both a spatial extent and formation time because a single total phase value cannot uniquely determine a pair of additive phases. Fortunately, we have used a broadband version of picosecond acoustic interferometry ${ }^{34,40,41}$ and acquired a full octave of acoustic bandwidth, where a tilt is clearly present in the $\mathrm{CC}$ phase spectrum in fig. 5B. Because opposite tilts are present in the individual spatial and temporal phase contributions (fig. S13), the tilt 
provides the additional information required to uniquely determine the spatial extent and formation time without constraining one or the other.

With these implications in mind, we performed a weighted least-squares fit of eq. $3 \mathrm{~b}$ on the $\mathrm{OC}$ and $\mathrm{CC}$ CAWs phase data shown in fig. $4 \mathrm{~b}$ with the spatial extent $(\xi)$ and formation time $\left(t_{0}\right)$ as the only free parameters. Figure $4 \mathrm{~b}$ shows the best fit curves with black dashed lines. For $\mathrm{CC}$, the best fit corresponded to a tensile strain with a formation time of $1.31 \pm 0.03 \mathrm{ps}$ and a spatial extent that converged at the $100 \pm 195 \mathrm{~nm}$ upper bound. For OC, we were unable to get a reliable fit because of the spatial and temporal phases' additive property combined with the larger spread in the OC data. The best fit corresponded to a compressive strain with a formation time that converged at the $0 \mathrm{ps}$ lower bound and a spatial extent of $6 \mathrm{~nm}$ with standard error of $1.5 \times 10^{4} \mathrm{~nm}$. From this, we can only conclude that the OC strain forms within the pump pulse duration. Note that the uncertainties we report are linearized standard errors and are unlikely to be accurate estimates of the true standard error, especially for the spatial extents because of its asymptotic relationship with phase. Nevertheless, the order of magnitudes of the standard errors strongly suggest that the extracted CC strain formation time is robust. More importantly, our model independently extracts the strain formation time, and this formation time is in quantitative agreement with the fast emissive growth rate as fit by eq. 1 (fig. S6).

Although we could not provide a quantitative estimate for the strain's spatial extent, we can estimate a lower bound by assuming that the strain formation time is the same as the fast emissive rise time as extracted by eq. 1 . In fig. $5 \mathrm{~A}$, we used the exponential fits to the $\mathrm{CC}$ emissive rise time (fig. S6) to constrain the $\mathrm{CC}$ phase to the grey box $\left(1.2 \pm 0.3 \mathrm{ps}, 150 \pm 4^{\circ}\right)$, where the $\mathrm{CC}$ average phase was calculated from the average phase of the $1.31 \mathrm{ps}$ fit and the bounds represent the standard deviation of the residuals. The corners of this box estimate the uncertainty in the spatial extent when using an independent measure of the interfacial strain's formation time from the fast emissive fit in eq. 1. To connect this box to the CAWs phase spectrum, we plot in fig. 4B the model phase curves for a $0.9 \mathrm{ps}$ formation time, $100 \mathrm{~nm}$ spatial extent and a $1.5 \mathrm{ps}$ formation time, $100 \mathrm{~nm}$ spatial extent, which represent the upper left and lower left corners on the box, respectively. This gives a visual understanding of the fitting quality of the 1.31 ps best fit phase curve, which is the target in the center of the box. In addition, we provide several other best fit curves with various constrained spatial extents in fig. S14B. When we constrained the formation time to $1.2 \mathrm{ps}$ in the model, we found the best fit spatial extent to be $12 \mathrm{~nm}$. The lower left corner 
of the box represents a lower bound estimate for the spatial extent, which we found to be $3.4 \mathrm{~nm}$ when we constrained the formation time to $0.9 \mathrm{ps}$. Visually, this lower bound represents a reasonable lower bound error based on the tilt shown by the black dashed curve in fig. S14B. In addition, in fig. S14A, we have generated curves to show how the best fit spatial extent varies asymptotically with different constrained formation times ranging from 0.9 to $1.33 \mathrm{ps,} \mathrm{which}$ demonstrates why we cannot assign an upper bound to the spatial extent through this model.

To find the strain magnitudes, we turn to the CAWs amplitude spectra shown in fig. $4 \mathrm{C}$. Because the relationship between the strain magnitude and CAWs amplitude depends on both the formation time and spatial extent, we constrained the spatial extents and formation times with the values described above and had the strain magnitude as a free parameter. For $\mathrm{pH} 13, \mathrm{CC}$ at 0.04 $\mathrm{mJ} / \mathrm{cm}^{2}$, the best fit strain magnitude was $0.06 \%$ for $1.2 \mathrm{ps}$ and $12 \mathrm{~nm}$. We estimate an upper bound for this strain to be $0.6 \%$ for $0.9 \mathrm{ps}$ and $3.4 \mathrm{~nm}$ and an asymptotic lower bound of $0.02 \%$ for 1.31 ps and $100 \mathrm{~nm}$. The dependance of strain magnitude on spatial extent is summarized in fig. S14A. Note that the CAWs amplitude spectra is fit to eq. 3a and includes a model for elasto-optic dispersion, $\Delta \epsilon_{x x}(\lambda) / \Delta \varepsilon_{z z}$, which is defined in eqs. S17 and S18. ${ }^{57}$ Figure S14C shows that the shape of the amplitude spectrum is insensitive to the spatial extent and formation time and is largely determined by this elasto-optic dispersion (fig. S12).

For $\mathrm{pH} 13 \mathrm{OC}$ at $2.0 \mathrm{~mJ} / \mathrm{cm}^{2}$, we used the pump intensity penetration depth of $15.5 \mathrm{~nm}$ (fig. S9B) and formation time of 0 ps to find a strain magnitude of $0.14 \%$. To compare the strain magnitude between OC and CC, we must normalize for the fluence. For OC this yields a strain of $0.07 \%$ per $\mathrm{mJ} / \mathrm{cm}^{2}$ and for CC a strain of $1.5 \%$ per $\mathrm{mJ} / \mathrm{cm}^{2}$ for the $12 \mathrm{~nm}$ estimate. As a comparison, thermal expansion would create a tensile strain magnitude of $0.08 \%$ per $\mathrm{mJ} / \mathrm{cm}^{2}$ with a $15.5 \mathrm{~nm}$ pump penetration depth (see section S15). ${ }^{55}$ Note that the magnitude agreement between OC strain and thermal strain is coincidental because thermal strain is tensile while our results indicate that OC strain is compressive. This sign difference suggests that the OC strain generation mechanism is related to, but not limited to, carrier deformation coupling and is caused by large changes in carrier densities. Finally, this comparison indicates that the CC strain magnitude is unusually strong, with a fluence normalized strain that is more than 20 times stronger than routine pumpinduced processes, such as thermal expansion.

\section{Discussion}



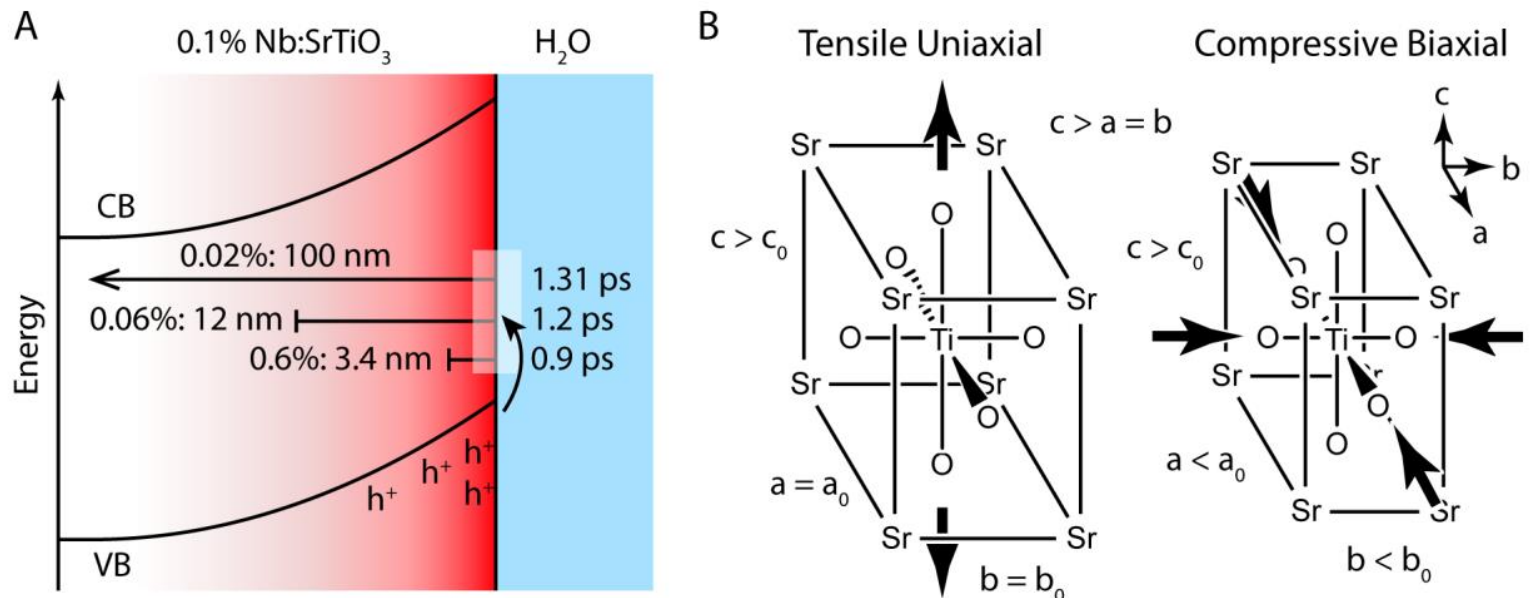

Figure 6: A) Graphical summary of the CC strain magnitudes, spatial extents, and formation times, as fit and estimated by the CAWs model. The red gradient represents the decay of the interfacial strain. The transparent white box indicates the mid-gap states. As a comparison, the pump intensity penetration depth is $15.5 \mathrm{~nm}$ and the depletion width is $\sim 25 \mathrm{~nm} .{ }^{9}$ B) A comparison of how the $\mathrm{TiO}_{6}$ octahedra are distorted under tensile uniaxial and out-of-plane strain (left, our work) and compressive biaxial and in-plane strain (right, strain engineering via lattice mismatch). ${ }^{58}$ The arrows indicate the direction of expansion and contraction. Although the volume of the unit cell changes, in both cases the strained crystal $c$-axis is larger than the $a$-and $b$-axis.

As summarized in fig. 6A, in $\mathrm{CC}$ we have tensile strain that forms with a $1.31 \mathrm{ps}$ time constant and an estimated a lower bound spatial extent of the strain to be $3.4 \mathrm{~nm}$. The tensile strain magnitude has a range between $0.02 \%$ to $0.6 \%$, with a best fit magnitude of $0.06 \%$. We assign this strain to hole-polaron trapping at the surface because of the strong correlations present for the formation time and amplitude. For OC, the strain was compressive, formed within the pump pulse duration, and correlated with the carrier population; we therefore assign it to carrier deformation coupling. The remainder of the discussion will concentrate on the $\mathrm{CC}$ strain. We first place the strain found in the $\mathrm{CC}$ conditions in the context of strain engineering, based on its upper bound and that it is tensile. We next consider the generation mechanisms by which hole-polarons could induce a similar interfacial strain. Finally, we discuss the possible origins of the 1.31 ps formation time.

The $0.6 \%$ upper bound tensile strain is similar in magnitude to strains generated in the field of strain engineering by lattice mismatch in epitaxial thin films ${ }^{20}$ For epitaxial thin films, the strain is typically biaxial and in-plane (001 and 010), whereas in this work the strain is uniaxial and outof-plane (100). The two types of strain can be compared by the unit cell's tetragonality, ${ }^{58}$ which measures the ratio of the unit cell's $c$ - (out-of-plane) to $a$ - and $b$-axis (in-plane). This is possible 
because strain introduces Jahn-Teller like distortions to the $\mathrm{TiO}_{6}$ octahedra, which leads to $e_{g}$ orbital splitting into $d_{x^{2}-y^{2}}$ and $d_{z^{2}}$ orbitals, whose energy ordering depends on the magnitude of tetragonality, and modifies $\mathrm{Ti} 3 \mathrm{~d}$ orbital filling. ${ }^{58}$ As shown in fig. 6B, our tensile out-of-plane strain correlates with compressive in-plane strain $(c>a)$.

While in strain engineering the lattice mismatch to the substrate is encoded in the growth, strain generation mechanisms of surface hole-polarons exist that could give rise to a continuum, tensile strain of a similar magnitude. In the classic Fröhlich (large polaron) ${ }^{59}$ and Holstein (small polaron $)^{60}$ descriptions, charge trapping is facilitated by optical phonons, ${ }^{61}$ which do not change the unit cell size and therefore does not directly induce strain. For polarons to induce strain, there must be coupling to acoustic phonons, either through electron-acoustic phonon coupling ${ }^{62-64}$ or through optical-acoustic phonon coupling. ${ }^{32}$ Two mechanisms exist for the electron-phonon case in particular: through the deformation potential to form an acoustic polaron, ${ }^{63}$ and through the piezoelectric effect to form a piezoelectric polaron. ${ }^{64}$ Normally, piezoelectricity is symmetry forbidden in room temperature STO because it is centrosymmetric, but the recent discovery of a surface piezoelectric effect induced by interfacial electric fields ${ }^{65}$ and surface relaxation ${ }^{66}$ indicates that such an effect is present, possibly to a large extent, at the STO/water interface. It is also worth noting that such a surface piezoelectric effect has been associated with perovskite octahedra rotation, ${ }^{67}$ which could reduce the effects of strain on OER activity. ${ }^{21}$

Other than deformations directly associated with the hole polaron, ${ }^{44}$ changes in free carrier concentrations can also contribute to interfacial strain. Under CC conditions, VB holes are the dominant free carrier near the surface because of the maintained Schottky barrier and resulting electron drift away from the surface (fig. S11). ${ }^{9}$ Under these conditions, the most likely mechanisms for free carrier strain generation are either through carrier deformation coupling ${ }^{31}$ or through an inverse-piezoelectric effect ${ }^{46}$ caused by VB hole accumulation near the surface and the aforementioned surface piezoelectric effect. ${ }^{65}$ We speculate that, if VB holes do significantly contribute to the strain, it would be dominated by the inverse-piezoelectric effect because carrier deformation coupling is likely weak, as shown by the comparatively weak OC strain. While the above mechanisms are possible, we emphasize that the strong correlation of strain with the holepolaron population suggests that polaronic distortion is the primary driver.

We now turn to the effects of our interfacial strain on OER catalysis and surface degradation. For OER/ORR catalysis, perovskite strain engineering typically uses a descriptor 
approach to correlate strain with oxygen adsorbate binding energies. ${ }^{20,22,68,69}$ This, combined with the universal scaling relations can correlate strain with OER activity. ${ }^{5,70}$ For our system, tensile uniaxial strain correlates with compressive biaxial strain, which favors $d_{z^{2}}$ orbital filling and therefore $e_{g}$ orbital filling, which is correlated with a weakening of the terminal Ti-O bond. ${ }^{21,71}$ This can be rationalized with a geometric argument: the $d_{z^{2}}$ orbital overlaps with the adsorbate oxygen $2 \mathrm{p}$ orbital in a $\sigma^{*}$ type bonding, therefore greater $d_{z^{2}}$ filling weakens the bond. ${ }^{71}$ Given that STO is a weak-binding catalyst, ${ }^{4,72}$ where OER is limited by a high $\mathrm{M}-\mathrm{OH}^{*}$ binding energy, ${ }^{5}$ we would expect a reduction in catalytic activity. Note that in our experiment, the thermionically limiting step of $\mathrm{M}-\mathrm{OH}^{*}$ formation causes the strain, and therefore the strain shifts the energy levels of forming additional $\mathrm{M}-\mathrm{OH}^{*}$ intermediates. It is also worth noting that, in liquids, a strain induced change in binding energies does not always translate to a change in catalytic activity. ${ }^{21}$

This descriptor approach has also been used to correlate strain with perovskite $\mathrm{ABO}_{3} \mathrm{~A}$ and B-atom vacancy formation energies, which have implications for surface degradation and amorphization. ${ }^{25}$ These trends are important because strain may facilitate the surface degradation present in our experiment (fig. S5). Note that surface degradation is also thought of as a kinetically hindered phase transition to a different region on a Pourbaix diagram induced by hole accumulation near the surface. ${ }^{23,27}$

Finally, the most striking result from this work is the quantitative agreement between the 1.3 ps CC strain and fast emissive growth rate. Previous work has established that the emissive component is a spectroscopic signature of hole-polaron formation at the surface (as a terminal or bridged oxyl species) and, at $0.04 \mathrm{~mJ} / \mathrm{cm}^{2}$, occurs with a 1.3 ps time constant. ${ }^{8}$ This time constant is also characteristic of the formation of an infrared active subsurface vibration, which is caused by lattice distortions associated with the terminal oxyl. ${ }^{12}$ The CAW identification of the 1.3 ps time constant extends the characterization of hole-polaron formation to the $\mathrm{GHz}$, acoustic domain. Namely, the formation of the hole-polaron's electronic states are tracked by visible emission, its vibrational states by mid-infrared normal modes, and the continuum, interfacial strain of a surface population by $\mathrm{GHz}$ phonons. The fact that the $1.3 \mathrm{ps}$ time constant is common to probes across the electromagnetic spectrum that definitively assigns it to a hole-trapping free energy with an elevated, but common temperature of the quantum states. Further, it suggests to which low energy surrounding modes that free energy dissipates. That hole-polaron formation occurs with an interfacial strain field directly implicates the propagating acoustic pulse in the dissipation. ${ }^{43,73}$ On 
the other hand, upon subtraction of the lattice phonons in the data, one is left with a GHz mode resulting from Brillouin scattering in the electrolyte (fig. S7), implicating the rotational-vibrational modes of water. Further, the 1.3 ps time constant itself has been assigned to the dissipation of an excited O-H stretch mode of water at interfaces. ${ }^{8,74-76}$ While the activation free energy could involve all these modes, and the 1.3 ps could be rate limited by any of one of them, the results show that the hole-trapping event to create OER intermediates in photo-electrochemistry implicates dissipation to both sides of the solid-liquid interface.

\section{Conclusion}

In this work, we found that ultrafast UV excitation of $0.1 \% \mathrm{Nb}: \mathrm{SrTiO}_{3}$ generates an interfacial strain at the $\mathrm{SrTiO}_{3}$-water interface that leads to the observed CAWs in our broadband TR spectra. Under CC (OER) conditions, a uniaxial (001) tensile strain is generated which we have assigned to the formation of the first OER intermediate, a hole-polaron trapped at surface oxygen sites. In OC conditions, the strain generation is compressive, 20 times weaker when normalized by pump fluence, and is likely caused by carrier-deformation coupling. For CC, we were able to quantitatively account for the CAWs phase and amplitude spectrum, which allowed us to extract a 1.3 ps formation time for the CC strain and estimate a lower bound spatial extent for the strain to be $3.4 \mathrm{~nm}$ with an upper bound strain magnitude to be $0.6 \%$. These results highlight some of the transient effects present under OER conditions that may perturb the OER mechanism and could be involved in the observed surface degradation. Finally, this work provides a more complete spectroscopic characterization of the first OER intermediate in $\mathrm{SrTiO}_{3}$ that now spans from the

$\mathrm{GHz}$ through to the UV-VIS. The distinctive $1.3 \mathrm{ps}$ formation time coincident with dissipation to low energy modes motivates future work on the dynamics behind hole-trapping, a critical step in any photo-driven electrochemical process.

\section{Acknowledgments}

The experimental work were supported by the Director, Office of Science, Office of Basic Energy Sciences, and by the Division of Chemical Sciences, Geosciences and Biosciences of the U.S. Department of Energy at RASEI (Boulder, CO) under Contract No. DE-SC0018939. This included partial (50\%) support for one research associate and for one graduate student. The experimental work was also supported by the Director, Office of Science, Office of Basic Energy Sciences, and by the Division of Material Sciences of the U.S. Department of Energy at SLAC 
under Contract No. DE-SC18050129 for RASEI (Boulder, CO). This supported one full graduate student.

\section{References:}

1. Z. W. Seh, J. Kibsgaard, C. F. Dickens, I. Chorkendorff, J. K. Norskov, and T. F. Jaramillo, "Combining theory and experiment in electrocatalysis: Insights into materials design," Science 355 (2017).

2. B. Hammer and J. K. Norskov, "Theoretical surface science and catalysis--calculations and concepts," Advances in Catalysis 45, 71 (2004).

3. N. M. Markovic, "Electrocatalysis: Interfacing electrochemistry," Nat. Mater. 12, 101 (2013).

4. M. Busch, N. B. Halck, U. I. Kramm, S. Siahrostami, P. Krtil, and J. Rossmeisl, "Beyond the top of the volcano? - A unified approach to electrocatalytic oxygen reduction and oxygen evolution," Nano Energy 29, 126 (2016).

5. J. Rossmeisl, Z. W. Qu, H. Zhu, G. J. Kroes, and J. K. Nørskov, "Electrolysis of water on oxide surfaces," Journal of Electroanalytical Chemistry 607, 83 (2007).

6. A. Vojvodic and J. K. Nørskov, "Optimizing Perovskites for the Water-Splitting Reaction," Science 334, 1355 (2011).

7. B. Klahr, S. Gimenez, F. Fabregat-Santiago, J. Bisquert, and T. W. Hamann, "Photoelectrochemical and Impedance Spectroscopic Investigation of Water Oxidation with "Co-Pi"-Coated Hematite Electrodes," Journal of the American Chemical Society 134, 16693 (2012).

8. X. Chen, S. N. Choing, D. J. Aschaffenburg, C. D. Pemmaraju, D. Prendergast, and T. Cuk, "The Formation Time of $\mathrm{Ti}-\mathrm{O} \bullet$ and $\mathrm{Ti}-\mathrm{O} \bullet-\mathrm{Ti}$ Radicals at the $\mathrm{n}-\mathrm{SrTiO} /$ Aqueous Interface during Photocatalytic Water Oxidation," Journal of the American Chemical Society 139, 1830 (2017).

9. M. M. Waegele, X. Chen, D. M. Herlihy, and T. Cuk, "How Surface Potential Determines the Kinetics of the First Hole Transfer of Photocatalytic Water Oxidation," Journal of the American Chemical Society 136, 10632 (2014).

10. F. L. Formal, E. Pastor, D. Tilley, C. A. Mesa, S. R. Pendlebury, M. Gratzel, and J. R. Durrant, "Rate Law Analysis of Water Oxidation on a Hematite Surface," J. Am. Chem. Soc. 137, 6629 (2015).

11. A. Kafizas, Y. Ma, E. Pastor, S. R. Pendlebury, C. Mesa, L. Francàs, F. Le Formal, N. Noor, M. Ling, C. Sotelo-Vazquez, C. J. Carmalt, I. P. Parkin, and J. R. Durrant, "Water Oxidation Kinetics of Accumulated Holes on the Surface of a TiO2 Photoanode: A Rate Law Analysis," ACS Catalysis 7, 4896 (2017).

12. D. M. Herlihy, M. M. Waegele, X. Chen, C. D. Pemmaraju, D. Prendergast, and T. Cuk, "Detecting the oxyl radical of photocatalytic water oxidation at an $\mathrm{n}$-SrTiO3/aqueous interface through its subsurface vibration," Nature Chemistry 8, 549 (2016).

13. M. Zhang, M. de Respinis, and H. Frei, "Time-resolved observations of water oxidation intermediates on a cobalt oxide nanoparticle catalyst," Nat Chem 6, 362 (2014).

14. M. Zhang and H. Frei, "Towards a Molecular Level Understanding of the Multi-Electron Catalysis of Water Oxidation on Metal Oxide Surfaces," Catalysis Letters 145, 420 (2014).

15. O. Zandi and T. W. Hamann, "Determination of photoelectrochemical water oxidation intermediates on haematite electrode surfaces using operando infrared spectroscopy," Nat. Chem. 8, 778 (2016).

16. J. Cheng, J. VandeVondele, and M. Sprik, "Identifying Trapped Electronic Holes at the Aqueous TiO2 Interface," Journal of Physical Chemistry C 118, 5437 (2014).

17. A. Janotti, J. B. Varley, M. Choi, and C. G. Van de Walle, "Vacancies and small polarons in \$\{\mathrm\{SrTiO\}\}_\{3\}\$," Physical Review B 90, 085202 (2014). 
18. N. A. Deskins and M. Dupuis, "Intrinsic Hole Migration Rates in TiO2 from Density Functional Theory," The Journal of Physical Chemistry C 113, 346 (2009).

19. O. F. Schirmer, "O-bound small polarons in oxide materials," Journal of Physics: Condensed Matter 18, R667 (2006).

20. J. Hwang, Z. Feng, N. Charles, X. R. Wang, D. Lee, K. A. Stoerzinger, S. Muy, R. R. Rao, D. Lee, R. Jacobs, D. Morgan, and Y. Shao-Horn, "Tuning perovskite oxides by strain: Electronic structure, properties, and functions in (electro)catalysis and ferroelectricity," Materials Today 31, 100 (2019).

21. D.-Y. Kuo, C. J. Eom, J. K. Kawasaki, G. Petretto, J. N. Nelson, G. Hautier, E. J. Crumlin, K. M. Shen, D. G. Schlom, and J. Suntivich, "Influence of Strain on the Surface-Oxygen Interaction and the Oxygen Evolution Reaction of SrlrO3," The Journal of Physical Chemistry C 122, 4359 (2018).

22. S. A. Akhade and J. R. Kitchin, "Effects of strain, d-band filling, and oxidation state on the surface electronic structure and reactivity of 3d perovskite surfaces," The Journal of Chemical Physics 137, 084703 (2012).

23. R. H. Zhang, N. Dubouis, M. Ben Osman, W. Yin, M. T. Sougrati, D. A. D. Corte, D. Giaume, and A. Grimaud, "A Dissolution/Precipitation Equilibrium on the Surface of Iridium-Based Perovskites Controls Their Activity as Oxygen Evolution Reaction Catalysts in Acidic Media," Angewandte Chemie-International Edition 58, 4571 (2019).

24. B. J. Kim, X. Cheng, D. F. Abbott, E. Fabbri, F. Bozza, T. Graule, I. E. Castelli, L. Wiles, N. Danilovic, K. E. Ayers, N. Marzari, and T. J. Schmidt, "Highly Active Nanoperovskite Catalysts for Oxygen Evolution Reaction: Insights into Activity and Stability of Ba0.5Sr0.5Co0.8Fe0.2O2+delta and PrBaCo2O5+delta," Advanced Functional Materials 28 (2018).

25. B. H. Han, M. Risch, Y. L. Lee, C. Ling, H. F. Jia, and Y. Shao-Horn, "Activity and stability trends of perovskite oxides for oxygen evolution catalysis at neutral pH," Physical Chemistry Chemical Physics 17, 22576 (2015).

26. B. J. Kim, D. F. Abbott, X. Cheng, E. Fabbri, M. Nachtegaal, F. Bozza, I. E. Castelli, D. Lebedev, R. Schaublin, C. Coperet, T. Graule, N. Marzari, and T. J. Schmidt, "Unraveling Thermodynamics, Stability, and Oxygen Evolution Activity of Strontium Ruthenium Perovskite Oxide," Acs Catalysis 7, 3245 (2017).

27. F. M. Toma, J. K. Cooper, V. Kunzelmann, M. T. McDowell, J. Yu, D. M. Larson, N. J. Borys, C. Abelyan, J. W. Beeman, K. M. Yu, J. H. Yang, L. Chen, M. R. Shaner, J. Spurgeon, F. A. Houle, K. A. Persson, and I. D. Sharp, "Mechanistic insights into chemical and photochemical transformations of bismuth vanadate photoanodes," Nature Communications 7 (2016).

28. C. Thomsen, H. T. Grahn, H. J. Maris, and J. Tauc, "Surface generation and detection of phonons by picosecond light pulses," Physical review. B, Condensed matter 34, 4129 (1986).

29. V. E. Gusev and P. Ruello, "Advances in applications of time-domain Brillouin scattering for nanoscale imaging," Applied Physics Reviews 5, 031101 (2018).

30. K. L. Pollock, H. Q. Doan, A. Rustagi, C. J. Stanton, and T. Cuk, "Detecting the Photoexcited Carrier Distribution Across GaAs/Transition Metal Oxide Interfaces by Coherent Longitudinal Acoustic Phonons," The Journal of Physical Chemistry Letters 8, 922 (2017).

31. P.-A. Mante, C. C. Stoumpos, M. G. Kanatzidis, and A. Yartsev, "Electron-acoustic phonon coupling in single crystal $\mathrm{CH} 3 \mathrm{NH} 3 \mathrm{Pbl} 3$ perovskites revealed by coherent acoustic phonons," Nature Communications 8, 14398 (2017).

32. M. Willatzen and Z. L. Wang, "Unified treatment of coupled optical and acoustic phonons in piezoelectric cubic materials," Physical Review B 92, 224101 (2015).

33. I. J. Chen, P.-A. Mante, C.-K. Chang, S.-C. Yang, H.-Y. Chen, Y.-R. Huang, L.-C. Chen, K.-H. Chen, V. Gusev, and C.-K. Sun, "Graphene-to-Substrate Energy Transfer through Out-of-Plane Longitudinal Acoustic Phonons," Nano Letters 14, 1317 (2014). 
34. S. Brivio, D. Polli, A. Crespi, R. Osellame, G. Cerullo, and R. Bertacco, "Observation of anomalous acoustic phonon dispersion in $\mathrm{SrTiO} 3$ by broadband stimulated Brillouin scattering," Applied Physics Letters 98, 211907 (2011).

35. D. J. Aschaffenburg, X. Chen, and T. Cuk, "Faradaic oxygen evolution from SrTiO3 under nano- and femto-second pulsed light excitation," Chemical Communications 53, 7254 (2017).

36. I. Vinogradov, S. Singh, H. Lyle, M. Paolino, A. Mandal, J. Rossmeisl, and T. Cuk. Measuring theoretical descriptors of the oxygen evolution reaction: Free energy difference to create $\mathrm{Ti}^{-} \mathrm{OH}^{*}$ by timeresolved optical spectroscopy. ChemRxiv, 2021; https://doi.org/10.26434/chemrxiv.13003490.v2

37. Y. Feng, I. Vinogradov, and N.-H. Ge, "General noise suppression scheme with reference detection in heterodyne nonlinear spectroscopy," Optics Express 25, 26262 (2017).

38. Y. Feng, I. Vinogradov, and N.-H. Ge, "Optimized noise reduction scheme for heterodyne spectroscopy using array detectors," Optics Express 27, 20323 (2019).

39. X. Chen, D. J. Aschaffenburg, and T. Cuk, "Selecting between two transition states by which water oxidation intermediates decay on an oxide surface," Nature Catalysis 2, 820 (2019).

40. S. Brivio, D. Polli, A. Crespi, R. Osellame, G. Cerullo, and R. Bertacco, "Response to "Comment on 'Observation of anomalous acoustic phonon dispersion in $\mathrm{SrTiO} 3$ by broadband stimulated Brillouin scattering'"'[Appl. Phys. Lett. 100, 206101 (2012)]," Applied Physics Letters 100, 206102 (2012).

41. A. Devos, Y.-C. Wen, P.-A. Mante, and C.-K. Sun, "Comment on "Observation of anomalous acoustic phonon dispersion in SrTiO3 by broadband stimulated Brillouin scattering" [Appl. Phys. Lett. 98, 211907 (2011)]," Applied Physics Letters 100, 206101 (2012).

42. Y. Lu, D. Jia, F. Gao, Z. Chen, and T. Hu, "First-principles study on the elastic properties of Sr-Ti-O ceramics," Solid State Communications 182, 43 (2014).

43. F. X. Morrissey and S. L. Dexheimer, "Coherent acoustic phonon generation in exciton self-trapping," Physical Review B 81, 094302 (2010).

44. B. Wu, W. Ning, Q. Xu, M. Manjappa, M. Feng, S. Ye, J. Fu, S. Lie, T. Yin, F. Wang, T. W. Goh, P. C. Harikesh, Y. K. E. Tay, Z. X. Shen, F. Huang, R. Singh, G. Zhou, F. Gao, and T. C. Sum, "Strong selftrapping by deformation potential limits photovoltaic performance in bismuth double perovskite," Science Advances 7, eabd3160 (2021).

45. C. Thomsen, H. T. Grahn, H. J. Maris, and J. Tauc, "Picosecond interferometric technique for study of phonons in the brillouin frequency range," Optics Communications 60, 55 (1986).

46. P. Babilotte, P. Ruello, T. Pezeril, G. Vaudel, D. Mounier, J.-M. Breteau, and V. Gusev, "Transition from piezoelectric to deformation potential mechanism of hypersound photogeneration in n-doped GaAs semiconductors," Journal of Applied Physics 109, 064909 (2011).

47. M. Daimon and A. Masumura, "Measurement of the refractive index of distilled water from the nearinfrared region to the ultraviolet region," Applied Optics 46, 3811 (2007).

48. M. N. Polyanskiy. Refractive index database https://refractiveindex.info; Vol. 2021.

49. O. Matsuda and O. B. Wright, "Laser picosecond acoustics in a two-layer structure with oblique probe light incidence," Ultrasonics 42, 653 (2004).

50. J. C. Bushnell and D. J. McCloskey, "Thermoelastic Stress Production in Solids," Journal of Applied Physics 39, 5541 (1968).

51. P. W. Voorhees and W. C. Johnson. The Thermodynamics of Elastically Stressed Crystals. In Solid State Physics; Ehrenreich, H., Spaepen, F., Eds.; Academic Press, 2004; Vol. 59; pp 1.

52. S. Adler, "Chemical Expansivity of Electrochemical Ceramics," Journal of the American Ceramic Society 84, 2117 (2001).

53. M. Tanaka, G. Girard, R. Davis, A. Peuto, and N. Bignell, "Recommended table for the density of water between $0 \mathrm{C}$ and $40 \mathrm{C}$ based on recent experimental reports," Metrologia 38, 301 (2001). 
54. N. Bilaniuk and G. S. K. Wong, "Speed of sound in pure water as a function of temperature," The Journal of the Acoustical Society of America 93, 1609 (1993).

55. D. de Ligny and P. Richet, "High-temperature heat capacity and thermal expansion of SrTiO3 and SrZrO3 perovskites," Physical review. B, Condensed matter 53, 3013 (1996).

56. J. Reintjes and M. B. Schulz, "Photoelastic Constants of Selected Ultrasonic Delay-Line Crystals," Journal of Applied Physics 39, 5254 (1968).

57. S. H. Wemple and M. DiDomenico, "Theory of the Elasto-Optic Effect in Nonmetallic Crystals," Physical Review B 1, 193 (1970).

58. D. Pesquera, G. Herranz, A. Barla, E. Pellegrin, F. Bondino, E. Magnano, F. Sánchez, and J. Fontcuberta, "Surface symmetry-breaking and strain effects on orbital occupancy in transition metal perovskite epitaxial films," Nature Communications 3, 1189 (2012).

59. H. Fröhlich, "Electrons in lattice fields," Advances in Physics 3, 325 (1954).

60. T. Holstein, "Studies of polaron motion: Part II. The "small" polaron," Annals of Physics 8, 343 (1959).

61. I. G. Austin and N. F. Mott, "Polarons in crystalline and non-crystalline materials," Advances in Physics 18, 41 (1969).

62. T. Higashimura, "Second-order energy shifts of the three types of bound polarons: the Frohlich, deformation potential and piezoelectric polarons," Journal of Physics C: Solid State Physics 20, 723 (1987).

63. F. M. Peeters and J. T. Devreese, "Acoustical polaron in three dimensions: The ground-state energy and the self-trapping transition," Physical Review B 32, 3515 (1985).

64. M. Engineer and G. Whitfield, "Strong-Coupled Piezoelectric Polaron," Physical Review 179, 869 (1969).

65. M.-M. Yang, Z.-D. Luo, Z. Mi, J. Zhao, S. P. E, and M. Alexe, "Piezoelectric and pyroelectric effects induced by interface polar symmetry," Nature 584, 377 (2020).

66. E. Meirzadeh, D. V. Christensen, E. Makagon, H. Cohen, I. Rosenhek-Goldian, E. H. Morales, A. Bhowmik, J. M. G. Lastra, A. M. Rappe, D. Ehre, M. Lahav, N. Pryds, and I. Lubomirsky, "Surface Pyroelectricity in Cubic SrTiO3," Advanced Materials 31, 1904733 (2019).

67. W. Kyung, C. H. Kim, Y. K. Kim, B. Kim, C. Kim, W. Jung, J. Kwon, M. Kim, A. Bostwick, J. D. Denlinger, Y. Yoshida, and C. Kim, "Electric-field-driven octahedral rotation in perovskite," npj Quantum Materials 6, 5 (2021).

68. J. Suntivich, K. J. May, H. A. Gasteiger, J. B. Goodenough, and Y. Shao-Horn, "A Perovskite Oxide Optimized for Oxygen Evolution Catalysis from Molecular Orbital Principles," Science 334, 1383 (2011).

69. A. Grimaud, K. J. May, C. E. Carlton, Y.-L. Lee, M. Risch, W. T. Hong, J. Zhou, and Y. Shao-Horn, "Double perovskites as a family of highly active catalysts for oxygen evolution in alkaline solution," Nature Communications 4, 2439 (2013).

70. J. H. Montoya, A. D. Doyle, J. K. Nørskov, and A. Vojvodic, "Trends in adsorption of electrocatalytic water splitting intermediates on cubic ABO3 oxides," Physical Chemistry Chemical Physics 20, 3813 (2018).

71. J. R. Petrie, V. R. Cooper, J. W. Freeland, T. L. Meyer, Z. Zhang, D. A. Lutterman, and H. N. Lee, "Enhanced Bifunctional Oxygen Catalysis in Strained LaNiO3 Perovskites," Journal of the American Chemical Society 138, 2488 (2016).

72. I. C. Man, H.-Y. Su, F. Calle-Vallejo, H. A. Hansen, J. I. Martínez, N. G. Inoglu, J. Kitchin, T. F. Jaramillo, J. K. Nørskov, and J. Rossmeisl, "Universality in Oxygen Evolution Electrocatalysis on Oxide Surfaces," ChemCatChem 3, 1159 (2011).

73. D. W. Brown, K. Lindenberg, and B. J. West, "On the dynamics of polaron formation in a deformable medium," The Journal of Chemical Physics 84, 1574 (1986). 
74. J. B. Asbury, T. Steinel, K. Kwak, S. A. Corcelli, C. P. Lawrence, J. L. Skinner, and M. D. Fayer, "Dynamics of water probed with vibrational echo correlation spectroscopy," The Journal of Chemical Physics 121, 12431 (2004).

75. J. B. Asbury, T. Steinel, C. Stromberg, S. A. Corcelli, C. P. Lawrence, J. L. Skinner, and M. D. Fayer, "Water Dynamics: Vibrational Echo Correlation Spectroscopy and Comparison to Molecular Dynamics Simulations," The Journal of Physical Chemistry A 108, 1107 (2004).

76. J. J. Loparo, S. T. Roberts, and A. Tokmakoff, "Multidimensional infrared spectroscopy of water. I. Vibrational dynamics in two-dimensional IR line shapes," The Journal of Chemical Physics 125, 194521 (2006). 